\title{
Transposable elements resistant to epigenetic resetting in the human germline are epigenetic hotspots for development and disease
}

\author{
Sabine Dietmann ${ }^{1,2,3,11 *}$, Michael J Keogh ${ }^{4,5,11}$, Walfred Tang ${ }^{2}$, Erna \\ Magnusdottir ${ }^{6}$, Toshihiro Kobayashi ${ }^{7,8}$, Patrick F Chinnery ${ }^{5,9}$ and M. Azim Surani \\ $1,2,10^{* * *}$
}

1. Wellcome Trust - Medical Research Council Stem Cell Institute, University of Cambridge, UK

2. Wellcome Trust/Cancer Research UK Gurdon Institute, University of Cambridge, Cambridge, UK

3. Department of Developmental Biology and Division of Nephrology, Washington University School of Medicine, St. Louis, MO, USA

4. Institute of Genetic Medicine, Newcastle University, Newcastle Upon Tyne, UK

5. MRC-Mitochondrial Biology Unit, University of Cambridge, Cambridge, UK

6. Department of Biomedical Science and Anatomy, Faculty of Medicine, University of Iceland, Reykjavik, Iceland

7. Section of Mammalian Transgenesis, Center for Genetic Analysis of Behavior, National Institute for Physiological Sciences, Okazaki, Japan

8. Department of Physiological Sciences, The Graduate University of Advanced Studies, Okazaki, Japan

9. Department of Clinical Neurosciences, Cambridge Biomedical Campus, University of Cambridge, Cambridge, UK

10. Department of Physiology and Development of Neuroscience, University of Cambridge, UK

11. These authors contributed equally

*Co-corresponding authors; sdietmann@wustl.edu; a.surani@gurdon.cam.ac.uk

** lead contact:a.surani@gurdon.cam.ac.uk 


\begin{abstract}
Despite the extensive erasure of DNA methylation in the early human germline, nearly eight percent of $\mathrm{CpGs}$ are resistant to the epigenetic resetting in the acutely hypomethylated primordial germ cells (week 7-9 hPGCs). Whether this occurs stochastically or represents relatively conserved layer of epigenetic information is unclear. Here we show that several predominantly hominoid-specific families of transposable elements (TEs) consistently resist DNA demethylation (henceforth called hPGC-methylated TEs or 'escapees') during the epigenetic resetting of hPGCs. Some of them undergo subsequent dynamic epigenetic changes during embryonic development. Our analysis of the fetal cerebral cortex also revealed multiple classes of young hPGC-methylated TEs within putative and established enhancers. Remarkably, specific hPGC-methylated TE subfamilies were associated with a multitude of adaptive human traits, including hair color and intelligence, and diseases including schizophrenia and Alzheimer's disease. We postulate that hPGC-methylated TEs represent potentially heritable information within the germline with a role in human development and evolution.
\end{abstract}




\section{INTRODUCTION}

The unique and comprehensive erasure of DNA methylation in early human primordial germ cells (hPGCs) during their migration into the developing gonads is a prerequisite for totipotency of zygotes, through which the germline transmits both genetic and epigenetic information for the development of subsequent generations. Recently, however, we identified TE subfamilies consisting primarily of hominoid-specific TEs, which are resistant to DNA demethylation during the epigenetic resetting leading to acute hypomethylation of hPGCs at $\sim$ week 7-9 of development ${ }^{1}$.

Approximately forty percent of the human genome consists of $\mathrm{TEs}^{2,3}$. Whilst older TEs have lost the ability to mobilise within the genome, young TEs can potentially retrotranspose, especially in the germline and during early development, when extensive epigenetic reprogramming occurs. To counter their activity, host defense mechanisms, including DNA methylation and piRNA biosynthesis, ensure their repression. The acute loss of DNA methylation in PGCs precedes the onset of piRNAs biosynthesis that could therefore potentially lead to the activation of TEs. Another mechanism for host defense might involve Krüppel-associated box (KRAB) zinc finger proteins (KZFPs) to suppress TEs in hPGCs. KRAB-ZFPs can bind to genomic retrotransposons, and transcriptionally silence them via the recruitment of repressive complexes through KAP1 ${ }^{4}$. In other contexts, such as during embryonic development, KZFP-TE interactions might influence the expression of surrounding genes ${ }^{4-6}$, and play a role in regulating development. Accordingly, there is a significant potential for the involvement of this large and rapidly evolving family of KZFPs during development ${ }^{7}$.

Approximately ninety-five percent of TEs have evolved neutrally from their ancestral origin $^{8}$, though some intergenic TEs appear to be under purifying selection for a functional role $^{9}$; for example, those located close to genes involved in human development ${ }^{10}$. Such positively selected TEs have undergone exaptation and acquire functions such as cis-regulatory elements (CREs) $)^{11,12}$, and recruit RNA polymerases to act as promotors ${ }^{13}$. Mutations within TEs can generate novel transcription factor (TF) binding sites ${ }^{14}$, and conversely, mutations in TFs can alter their functions. The acquisition of mutations within TFs and their binding sites, such as in TEs, might promote novel gene regulatory networks for for mammalian development ${ }^{15}$. Recent studies suggest that the interaction between KZFPs and TEs that have arisen within our 
hominoid ancestry might facilitate their development as cis regulators affecting early and late embryogenesis ${ }^{16}$. Accordingly, KZFP-TE mediated chromatin modifications have the potential to affect nearby genes within the developing fetus in tissue and cell-specific manner; this may account for species-specific epigenetic and transcriptional networks even when they share broadly similar genomes ${ }^{16},{ }^{17-19}$. What drives or promotes the formation of new gene regulatory networks remains to be fully elucidated.

Here we focus on the DNA demethylation-resistant hominoid-specific TEs in hPGCs and ask whether their presence within the germline might have contribute to human development. By considering the age at which incorporation of each TE occured into the human genome ${ }^{19}$ and their putative biological effects, we explore how they might have facilitated specific facets of human development. In particular, we have considered this possibility in the context of human neurological development.

\section{RESULTS}

\section{The spectrum of transposable elements resistant to DNA methylation erasure in hPGCs}

First, we examined the genomic regions that are resistant to DNA methylation erasure within hPGCs (in male and female week 7-9 hPGCs), when they reach an unprecedented acute hypomethylated state $^{1}$ (Fig. 1a). Using a hidden Markov model (HMM), we identified 116,617 significantly hypermethylated genomic regions, with average methylation of $>30 \% ; 60,657$ of them overlap with those from an independent study of week 10-11 hPGCs ${ }^{21}$ (Fig. 1b, Extended Data Fig.1a, Supplementary Table 1).

We detected 117,161 transposable elements within the hypermethylated genomic regions (subsequently termed 'hPGC-methylated TEs', Fig. 1c), many of which were hominoid-specific ( $<25$ million years old) and shared with apes. While a lower proportion of them was also present in macaques, a negligible proportion was found in distant mammals as compared to all TEs (Fig. 1d, Extended Data Fig. 1c, Supplementary Table 2). A smaller number of older TE families of $>25$ million years, however, also remained methylated (Extended Data Fig. 1b,c; Supplementary Table 2,3). 
The hPGC-methylation of TEs varies markedly by TE family, with Alu (AluY, AluSx; members of the SINE family), long-terminal repeat (LTR) THE1B, together with the long interspersed nuclear element (LINE) L1PA3-7 subfamilies of retrotransposons (Fig. 1b) being particularly abundant within hypermethylated regions. The proportion of TEs within these subfamilies that are methylated also appear to be proportional to the age of the TE subfamily. For example, in the hominid-specific subset of retrotransposons, SINE-VNTRAlu (SVA), SVA_A (the oldest SVA originating $\sim 15$ million years ago in early primate evolution $\left.^{22}\right)$ had a far higher percentage of methylated elements $(\sim 80 \%)$, compared to the much younger human-specific SVA_E (3.5 million years old ${ }^{22}$ ), with only $\sim 25 \%$ of methylated elements (Extended Data Fig. 1b,c). Similarly, in LINE1 family, where L1PA3-7 are between 12.5 and 31 million years old, older TEs (e.g. L1PA7) show a greater proportion of TEs remaining methylated compared to younger TEs (e.g. L1PA3). This is observed even though all these TE subfamilies are inactive in humans ${ }^{22}$. Other very young TEs, such as multiple members of the AluY family, which arose $<3$ million years ago within humans ${ }^{24}$, remain largely unmethylated even though they have the potential to mobilize (Extended Data Fig. 1b,c).

Why a progressive increase in DNA methylation occurs with the age of TEs, and whether it has any functional significance is unknown. However, it suggests that the presence of hPGC-methylation of TEs may not primarily function to reduce TE activity, as transposition activity decreases with TE age. Since the acute DNA demethylation of week 7-9 hPGCs occurs before the onset of Piwi-RNAs (piRNA) ${ }^{25-27}$, (another key host defense mechanism), implicates other mechanisms, such as histone modifications to counter potential TE activity. In this regard, KZFP-TE interactions also merit consideration for regulating potentially active and very young TEs ( $<1$ million years old) during the hypomethylation of week 7-9 hPGCs (see below).

\section{Tracing the progression of hPGC-methylated TEs at the onset of development}

To begin to determine whether hPGC-methylation of TEs may therefore function to promote histone modifcations within human tissues (rather than repress TE activity within the germline), we determined the overlap between hPGC methylated TEs and multiple histone modifications present within 37 primary tissues representative of all significant human lineages ${ }^{28}$. We observed that hPGC-methylated TEs intersected 
H3K9me3 marks significantly more frequently (58\%) than non-methylated TEs $(25 \%)$ in the genome ( $P$ value $<2 e-16$, Pearson's Chi-squared test) (Fig. 2a). The enrichment of H3K9me3 marks in hPGC-methylated TEs appears to arise at different rates between different TE subfamilies. For example, hPGC-methylated SVAs are enriched for fetal H3K9me3 marks within 3.2 million years (SVA_F), whereas the youngest LINE1 hPGCmethylated TEs acquire $\mathrm{H} 3 \mathrm{~K} 9$ me3 marks within $\sim 7.6$ million years ${ }^{29}$ (Fig. 2b, Extended Data Fig. 2a). Importantly, these timeframes also reflect the rate at which DNA methylation is acquired within hPGCs by these families (Fig. 2c). We also observe an enrichment of $\mathrm{H} 3 \mathrm{~K} 9 \mathrm{me} 3$ on human-specific hPGC-methylated TEs in the fetal and adult brain (see below, Fig. 2b, Extended Data Fig. 2a). These data further support the hypothesis that hPGC-methylated TEs may mark or even influence the acquisition of H3K9me3 in human tissues. Moreover, some of the TE subfamilies with the highest fraction of hPGC-methylated elements, including LTR12, MER11A-B. LTR12C, and the SVAs, were significantly enriched for tissue-specific enhancer marks (K4me1+K27ac) ( $P$ value $<1 \mathrm{e}-5$, Pearson's Chi-squared test, Extended Data Fig. 2b, Supplementary Table 4).

To try and gain insigt into how hPGC-methylation may influence the potential development of both $\mathrm{H} 3 \mathrm{~K} 9 \mathrm{me} 3$ and enhancer histone modifications, we considered the fate of hPGC-methylated TEs during subsequent development. Beginning with gametogenesis, we observed that the majority of older hPGC-methylated TEs retain methylation during gametogenesis in a relatively homogeneous fashion. The youngest TEs however (including AluYa5, AluYg6, LTR12C-F, and SVA B-F), showed significant DNA methylation heterogeneity within oocytes, and more markedly within sperm (Extended Data Fig. 3a-c, Supplementary Table 2,5). Consequently, some young TE subfamilies may be a source of epigenetic heterogeneity in sperm, while older methylated TEs are likely to be conserved and consistently transmitted by sperm and oocytes.

Since it is technically challenging to follow the fate of hPGC-methylated regions during early human development, we considered a surrogate model of in vitro transition of human embryonic stem cells (hESCs) from naïve to primed, representing the preimplantation epiblast, and the more advanced pre-streak gastrulating postimplantation epiblast, respectively ${ }^{30,-32}$ (Fig. 3a). We observed overall a correlation of 
DNA methylation levels in hPGC-methylated TEs between week 7-9 hPGCs and the preimplantation epiblast (Extended Data Fig. 3b); the hPGC-methylated regions showed distinct dynamic changes concerning $\mathrm{H} 3 \mathrm{~K} 9 \mathrm{me} 3$ and DNA methylation during the transition to primed hESCs. Evidently, some TEs such as SVA_D and SVA_F show marked increases in H3K9me3 levels in primed hESCs in both hPGC-methylated and non-methylated TEs. Conversely, we observed a loss of H3K9me3 on the hPGCmethylated elements of most TE subfamilies in primed hESCs, while there is a gain of H3K9me3 on unmethylated TEs (Fig. 3b,c, Extended Data Fig. 3d). A fraction (5-10\%) of hPGC-methylated TEs that lose H3K9me3 in primed hESCs intersects with established tissue-specific enhancer signatures (Fig. 3b,c), including TE elements of the LTR1D, LTR12C, HERV9 and HERVH subfamilies ${ }^{33,34}$. In contrast, hPGC-methylated TEs that gain H3K9me3 during the naïve-to-primed transition generally lack tissue-specific enhancer marks (Fig. 3b).

The loss of $\mathrm{H} 3 \mathrm{~K} 9 \mathrm{me} 3$ enables lineage selection in early progenitor cells, accompanied by more dynamic changes than was thought previously ${ }^{35}$. Observations of early murine embryos, for example, have also shown that $\mathrm{H} 3 \mathrm{~K} 9 \mathrm{me} 3$ may replace DNA methylation on TEs in a family-specific manner during preimplantation development ${ }^{36}$. H3K9me3 in the preimplantation epiblast, with its widespread DNA hypomethylation, might therefore 'mark' these TEs before DNA re-methylation occurs in the postimplantation epiblast. Irrespective of whether $\mathrm{H} 3 \mathrm{~K} 9 \mathrm{me} 3$ is enriched or lost, methylation of TEs in hPGCs appears likely to influence the histone modification dynamics within TE families during naive to primed transition. Notably, a reduction in $\mathrm{H} 3 \mathrm{~K} 9 \mathrm{me} 3$ levels on the majority of TE families is associated with lineage-specific enhancers.

Thus, hPGC-methylation of TEs is strongly associated with the subsequent acquisition of $\mathrm{H} 3 \mathrm{~K} 9 \mathrm{me} 3$, as well as enhancer marks in human tissues. Notably, the initiation of the onset of lineage-specific histone marks therefore appears to begin in the germline with the retention of DNA methylation within hPGCs.

\section{TE-derived enhancers may facilitate the development of the human fetal brain}

Since the enrichment of several TE subfamilies occurs within brain-specific H3K9me3marked regions (see above, Fig. 2b, Extended Data Fig. 2a), we explored the role of 
hPGC-methylated TEs in the context of the human fetal brain. We re-analyzed data from the developing cortex ${ }^{37}$ and identified 29,679 syntenic regions, enriched for H3K27ac in both human and rhesus macaque, as putative active enhancers (Supplementary Table 6). The human brain has evolved markedly compared to the macaque, being approximately 4.8 times larger in size (adjusted for body weight) ${ }^{38}$, with a comparatively $35 \%$ larger neocortex ${ }^{39}$. We identified novel insertions of hPGC-methylated TEs in human within these orthologous enhancer regions. LTR12C was the most highly hPGCmethylated TE insertion found within human brain enhancers (Fig. 4a). Pathway analysis revealed an enrichment of LTR12C within genes controlling cell adhesion, neurogenesis, and astrocyte differentiation that are all associated with corticogenesis (Fig. 4c). Notably, LTR12C has been reported to be a primary source of novelty in primate gene regulation ${ }^{19}$, which can influence human corticogenesis. Accordingly, hPGC-methylation may be associated with the promotion of human specific enhancer formation within the developing brain and potentially other tissues.

For further support, we examined the relationship between TEs and the gene expression network of the fetal brain. We analysed 284 RNA-seq datasets from differing brain regions in the cortex and cerebellum of 172 fetal brains (post-conceptual week 4-17; mean 10.05 weeks) $)^{40}$. Identifying 20 unique modules in 6,766 differentially expressed genes $(\log 2 \mathrm{FC}>1, \mathrm{P}$ value $<0.05)$, we observed that ape-specific hPGC-methylated TEs were primarily enriched in cortically expressed modules compared to those in the cerebellum (Extended Data Fig. 4a,b, Supplementary Table 7); the latter is significantly less diverse, functionally and morphologically, in humans compared to the cortex ${ }^{41,42}$. Within module 5 , the most preferentially cortically expressed module, the insertion of two or more hPGC-methylated TEs within multiple nodes that correlate with gene expression (Fig 4d). These genes include EOMES expression in intermediate radial glial progenitor cells as they enter a proneural state ${ }^{43}$, and EMX1 that is critical for neocortical patterning ${ }^{44}$. Together they are significant for the development of human cortical gyrification, which is an adaptive evolutionary process in mammals facilitating cognitive development ${ }^{45}, 46$. In another cluster, DMRTA2 and DLX2 are both associated with at least one hPGC-methylated TE, and these genes are involved in neuronal migration and the switch between neuronal and oligodendrocyte cell fate, respectively ${ }^{47,48}$. 
Together, these observations show that hPGC-methylated regions exist at sites associated with networks that shape components of the unique architecture of the human brain, and within enhancers of cortical development, and gene-expression modules active in human neurodevelopment.

\section{The potential impact of hPGC-methylated TEs in the human cortex}

Genes within Module 5 were more highly expressed in the developing human cortex than in chimps (Fig. 4d,e), prompting us to investigate several facets of hPGCmethylated and non-methylated TE expression in this context. Comparing 284 RNA-seq datasets from differing brain regions in the cortex, cerebellum and midbrain of 172 fetal brains (275 samples, see above), we observed 10,346 expressed TE elements (irrespective of DNA methylation status in PGCs), which exhibited clear spatiotemporal dynamic expression (Extended Data Fig. 4c, PC2 vs. PC1 and PC4 vs PC3). Young hPGC-methylated TEs (those shared with great apes or 'ape-specific TEs') such as L1PA2 ( 7.6 million years old) showed high cortical expression, compared to L1PA4-11 families $\left(\sim 13\right.$ to 53 million years old $\left.{ }^{29}\right)$. Notably, the expression of the latter was preferentially observed within the cerebellum (Extended Data Fig. 4d). When comparing genes that are in close proximity $(<50 \mathrm{~kb})$ to all differentially expressed TEs $(\log 2 \mathrm{FC}>2$ ), we observed that hPGC-methylated regions were strongly associated with critical developmental processes in comparison to non-methylated TEs (Fig. 4b). One notable example is provided by FOXP249, which has facilitated language development during human evolution ${ }^{49,50}$. Accordingly, these data further demonstrate that hPGC-methylated regions are strongly enriched within enhancers and gene-networks that are primarily active during corticogenesis, and importantly with those that show differential expression within the developing cortex.

\section{KRAB zinc finger proteins may mediate the exaptation of TEs as developmental enhancers}

Next, we determined potential mediators that influence the enhancer activity of hPGCmethylated TEs in the brain. In particular, we considered the role of KZFPs, which binding to TEs to repress their activity in human embryonic stem cells (ESCs) and the early embryo ${ }^{16,51}$. Through their ability to recruit KRAB-associated protein 1 (KAP1) complex, histone-deacetylase-containing NuRD complex, histone-lysine 
methyltransferase SETDB1 and DNA methyltransferases ${ }^{29}$, they also mediate methylation and chromatin modifying effectors ${ }^{29}$ (Extended Data Fig. 5a).

Analysis of genome-wide binding to TEs (ChIP-exo) for $236 \mathrm{KZFPs}^{7}$ showed that multiple KZFPs, including ZNF93 and ZNF534, retain preferential binding for hominoidspecfic methylated TEs (L1HS and L1PA2, and HERVH, Extended Data Fig. 5b). When considering the binding of KZFPs on all TEs (methylated and non-methylated) by age, we observed a clear propensity for KZFPs to bind to the majority of young TEs (L1HS L1PA3) irrespective of their methylation status, before subsequent continued enrichment of KZNFs binding to hPGC-methylated TEs (Extended Data Fig. 5c, Supplementary Table 8). It is possible that KZFPs may initially function primarily to suppress TE activity, but over time, KZFP-TE interactions may function to provide a layer of DNA methylation and possibly chromatin modifications at KZFP-TE sites. Although further experimental is required, it is possible that if such epigenetic modifications could then be transmitted to the totipotent zygote at fertilization, then this may offer a potential mechanism for intergenerational epigenetic inheritance.

After establishing KZFPs that are expressed in week 7-9 hPGCs and dynamically expressed in the brain, we found a subset of KZFPs with high expression within the cortex, including ZNF534, ZNF257 and ZNF98 (Fig. 5a). Moreover, we detected multiple examples of KZFP-TE interactions, where the expression of the KZFP in the cortex and cerebellum was significantly anti-correlated with that of the hPGC-methylated TEs, to which they have binding motifs, in particular for members of the LTR12 and SVA families (Fig. 5b, Supplementary Table 9). Some of these dynamically expressed hPGCmethylated TEs provides further evidence of KAP1 binding, and binding of the corresponding KZFP by ChIP-exo (Fig. 5c), We also found associated correlated expression of overlapping or near-by genes, which, as above, are enriched for those influencing neurodevelopmental processes; and this correlation was particularily strong for hPGC-methylated TEs with evidence for KZFP binding (Extended Data Fig. 5d). Thus, we propose that dynamically expressed KZFPs within the brain may bind to their corresponding TE motifs and influence localized gene expression, generating a somatically-acting exapted KZFP-TE network within the brain as observed in other cell types ${ }^{16}$. Crucially however, the onset of this process may begin within hPGCs and not 
entirely somatically; accordingly, the nidus for these networks may be inherited from the germline.

\section{Association of hPGC-methylated regions with human traits and diseases}

Finally, we examined if some TE subfamilies may be associated with common human diseases. Comparing the overlap between methylated TEs and genes identified in GWAS loci associated with human diseases and traits, we identified 294 TE subfamilies that were enriched for at least one human trait or disease (Supplementary Table 10), including height and intelligence, and diseases including schizophrenia and Alzheimer's disease (Fig. 5d). Particular TE classes were enriched for a heterogeneous array of traits or diseases affecting multiple organs, were families such as LTR12C enriched conditions including type 2 diabetes mellitus, oesophageal carcinoma and Alzheimer's disease. In contrast, other families were associated primarily with single organ conditions such as LTRA8, which was enriched for dermatological traits and conditions such as hair colour, vitiligo and sensitivity to sun (Extended Data Fig. 6a). These observations suggest that hPGC-methylated regions may be associated with mediating both adaptive traits and also potentially several human diseases. Altogether evidence suggests that hPGC-methylated regions are likely to have an impact on the regulation of genes that have significant functions in human health and disease. Speculatively, variations in hPGC methylation within specific TE families may constitute a risk factor for certain disorders, which may partly explain the elusive nature of heritability of some conditions and traits ${ }^{52}$.

\section{DISCUSSION}

Our study provides a foundation for testable hypotheses that the transgenerational inheritance of loci, which are resistant to the acute epigenetic-resetting in the human germline, might have a significant role in human evolution, development, and disease. It is critical to establish whether the persistent methylation of transposable elements within the human germline is a stochastic process, or an adaptive component of the human germline, which primarily involves hominoid-specific TEs ${ }^{1}$. The exact mechanism and mediators of resistance to DNA demethylation in the acutely hypomethylated hPGCs remain to be determined, but KZFPs binding is amongst a possibility to mediate this process $^{53}$. KZFPs binding to young TEs appears prevalent regardless of their DNA 
methylation status, as well as to older TEs that are no longer mobile. The apparent progressive increase in the proportion of TEs with methylation in various subfamilies is noteworthy. The basis for such an outcome merits consideration, together with the impact of the persistent TE-methylation and their transmission, and their implications for evolutionary biology, human development and disease.

The TE family-specific methylation reprogramming within gametes and the significant methylation in hPGCs correlate with reprogramming of H3K9me3. Their enrichment with heterochromatin marks in early embryonic stem cells and their in vivo counterpart may provide the nidus for the onset of somatic epigenetic programming during human development ${ }^{54}$. Notably, the timing of these events could coincide with the rapid periimplantation epiblast proliferation and selection through cell competition, before the onset of somatic and germ cell fates. For example, the marked association of methylated ERVs and LTRs (such as LTR12C or MER52A) with H3K9me3 erasure in primed ESCs, and whether KZFP binding at this point plays a role, remains to be established. Conversely, hPGC-methylated SVA elements, including very young human-specific elements such as SVA_E and SVA_F, which lack tissue-specific histone marks, show a strong increase of $\mathrm{H} 3 \mathrm{~K} 9 \mathrm{me} 3$ in primed pluripotent cells. Such behaviour in the early phase of development, where cells gain a competent state for all three germ layers and the germline, may ensure the co-association with $\mathrm{H} 3 \mathrm{~K} 9 \mathrm{me} 3$ in a relatively homogeneous fashion; subsequent selection processes may act to retain $\mathrm{H} 3 \mathrm{~K} 9 \mathrm{me} 3$ marks in a tissue specific manner.

A recent study suggested that Krüppel-like transcription factors, including KLF4 can activate the embryonic genome in early embryos through binding to young TEs and inducing their expression; subsequent expression of KZFPs then represses the TEs ${ }^{16}$. Similar events occur in naïve hESCs that contain relatively hypomethylated DNA compared to primed hESCs. Since at least some KLF family members are expressed in early human PGCs undergoing DNA demethylation ${ }^{55,56}$, this might suggest that a similar feedback mechanism could operate in the human germline. In addition, the most recently acquired ERV (HERVK) expression is also detected and driven by OCT4 in human preimplantation embryos that ceases after implantation ${ }^{57}$. The hominoid-specific young TEs in the germline, and their exaptation may provide species-specific regulators of gene expression in multiple tissues. 
We provide a potential basis for somatic modulation of hPGC-methylated TEs within the brain. Accordingly, young methylated TEs have become enriched within a variety of modules that can apparently influence neurodevelopmental processes, in particular cortical developmental networks. These networks incorporate both enhancers (such as LTR12C or SVAs) and TEs associated with repressive histone complexes such as H3K9me3. Within 5 million years of their incorporation into the human genome (based on genome age), we detect exapted somatic TE networks, which merits further investigation. With more precise information on TE loci within the genome, high quality cell-specific brain RNA-seq datasets could reveal their influence on human brain development as well as in other somatic tissues. An association between methylated TEs in almost 300 families and several human traits and diseases suggests that these TEs may influence a multitude of adaptive human traits across multiple organ systems and tissues together with several human diseases.

It remains a possibility that the hominoid-specific TE loci that are resistant to reprogramming and retain methylation in hPGCs, constitute a layer of heritable information, with an impact on human evolution, development and disease.

\section{References}

1. Tang WW, Dietmann S, Irie N, et al. A Unique Gene Regulatory Network Resets the Human Germline Epigenome for Development. Cell 2015; 161(6): 1453-67.

2. Lander ES, Linton LM, Birren B, et al. Initial sequencing and analysis of the human genome. Nature 2001; 409(6822): 860-921.

3. Venter JC, Adams MD, Myers EW, et al. The sequence of the human genome. Science 2001; 291(5507): 1304-51.

4. Wolf D, Goff SP. TRIM28 mediates primer binding site-targeted silencing of murine leukemia virus in embryonic cells. Cell 2007; 131(1): 46-57.

5. Ecco G, Cassano M, Kauzlaric A, et al. Transposable Elements and Their KRABZFP Controllers Regulate Gene Expression in Adult Tissues. Developmental cell 2016; 36(6): 611-23.

6. Wolf D, Goff SP. Embryonic stem cells use ZFP809 to silence retroviral DNAs. Nature 2009; 458(7242): 1201-4.

7. Imbeault M, Helleboid PY, Trono D. KRAB zinc-finger proteins contribute to the evolution of gene regulatory networks. Nature 2017; 543(7646): 550-4.

8. Meader S, Ponting CP, Lunter G. Massive turnover of functional sequence in human and other mammalian genomes. Genome Res 2010; 20(10): 1335-43. 
9. de Souza FS, Franchini LF, Rubinstein M. Exaptation of transposable elements into novel cis-regulatory elements: is the evidence always strong? Molecular biology and evolution 2013; 30(6): 1239-51.

10. Lowe CB, Bejerano G, Haussler D. Thousands of human mobile element fragments undergo strong purifying selection near developmental genes. Proceedings of the National Academy of Sciences of the United States of America 2007; 104(19): 800510.

11. Notwell JH, Chung T, Heavner W, Bejerano G. A family of transposable elements co-opted into developmental enhancers in the mouse neocortex. Nature communications 2015; 6: 6644.

12. Bejerano G, Lowe CB, Ahituv N, et al. A distal enhancer and an ultraconserved exon are derived from a novel retroposon. Nature 2006; 441(7089): 87-90.

13. Slotkin RK, Martienssen R. Transposable elements and the epigenetic regulation of the genome. Nat Rev Genet 2007; 8(4): 272-85.

14. Feschotte C. Transposable elements and the evolution of regulatory networks. Nat Rev Genet 2008; 9(5): 397-405.

15. Garcia-Perez JL, Widmann TJ, Adams IR. The impact of transposable elements on mammalian development. Development 2016; 143(22): 4101-14.

16. Pontis J, Planet E, Offner S, et al. Hominoid-Specific Transposable Elements and KZFPs Facilitate Human Embryonic Genome Activation and Control Transcription in Naive Human ESCs. Cell Stem Cell 2019; 24(5): 724-35 e5.

17. Mendizabal I, Shi L, Keller TE, et al. Comparative Methylome Analyses Identify Epigenetic Regulatory Loci of Human Brain Evolution. Molecular biology and evolution 2016; 33(11): 2947-59.

18. Erwin JA, Marchetto MC, Gage FH. Mobile DNA elements in the generation of diversity and complexity in the brain. Nature reviews Neuroscience 2014; 15(8): 497506.

19. Trizzino M, Park Y, Holsbach-Beltrame M, et al. Transposable elements are the primary source of novelty in primate gene regulation. Genome Res 2017; 27(10): 162333.

20. Malik HS, Burke WD, Eickbush TH. The age and evolution of non-LTR retrotransposable elements. Molecular biology and evolution 1999; 16(6): 793-805.

21. Guo F, Yan L, Guo H, et al. The Transcriptome and DNA Methylome Landscapes of Human Primordial Germ Cells. Cell 2015; 161(6): 1437-52.

22. Wang H, Xing J, Grover D, et al. SVA elements: a hominid-specific retroposon family. J Mol Biol 2005; 354(4): 994-1007.

23. Hancks DC, Kazazian HH, Jr. Active human retrotransposons: variation and disease. Curr Opin Genet Dev 2012; 22(3): 191-203.

24. Xing J, Hedges DJ, Han K, Wang H, Cordaux R, Batzer MA. Alu element mutation spectra: molecular clocks and the effect of DNA methylation. J Mol Biol 2004; 344(3): 675-82.

25. Lukic S, Chen K. Human piRNAs are under selection in Africans and repress transposable elements. Molecular biology and evolution 2011; 28(11): 3061-7.

26. Aravin AA, Hannon GJ, Brennecke J. The Piwi-piRNA pathway provides an adaptive defense in the transposon arms race. Science 2007; 318(5851): 761-4. 
27. Siomi MC, Sato K, Pezic D, Aravin AA. PIWI-interacting small RNAs: the vanguard of genome defence. Nat Rev Mol Cell Biol 2011; 12(4): 246-58.

28. Chadwick LH. The NIH Roadmap Epigenomics Program data resource. Epigenomics 2012; 4(3): 317-24.

29. Khan H, Smit A, Boissinot S. Molecular evolution and tempo of amplification of human LINE-1 retrotransposons since the origin of primates. Genome Res 2006; 16(1): 78-87.

30. Nichols J, Smith A. Naive and primed pluripotent states. Cell Stem Cell 2009; 4(6): 487-92.

31. Nichols J, Smith A. Pluripotency in the embryo and in culture. Cold Spring Harbor perspectives in biology 2012; 4(8): a008128.

32. Guo G, von Meyenn F, Rostovskaya M, et al. Epigenetic resetting of human pluripotency. Development 2017; 144(15): 2748-63.

33. Medstrand P, Landry JR, Mager DL. Long terminal repeats are used as alternative promoters for the endothelin $\mathrm{B}$ receptor and apolipoprotein C-I genes in humans. The Journal of biological chemistry 2001; 276(3): 1896-903.

34. Jern P, Coffin JM. Effects of retroviruses on host genome function. Annual review of genetics 2008; 42: 709-32.

35. Nicetto D, Donahue G, Jain T, et al. H3K9me3-heterochromatin loss at proteincoding genes enables developmental lineage specification. Science 2019; 363(6424): 2947.

36. Wang C, Liu X, Gao Y, et al. Reprogramming of H3K9me3-dependent heterochromatin during mammalian embryo development. Nature cell biology 2018; 20(5): $620-31$.

37. Reilly SK, Yin J, Ayoub AE, et al. Evolutionary genomics. Evolutionary changes in promoter and enhancer activity during human corticogenesis. Science 2015;

347(6226): 1155-9.

38. MacLeod CE, Zilles K, Schleicher A, Rilling JK, Gibson KR. Expansion of the neocerebellum in Hominoidea. Journal of human evolution 2003; 44(4): 401-29.

39. Rilling JK, Insel TR. The primate neocortex in comparative perspective using magnetic resonance imaging. Journal of human evolution 1999; 37(2): 191-223.

40. Martikainen MH, Chinnery PF. Mitochondrial disease: mimics and chameleons. Pract Neurol 2015; 15(6): 424-35.

41. Weaver AH. Reciprocal evolution of the cerebellum and neocortex in fossil humans. Proceedings of the National Academy of Sciences of the United States of America 2005; 102(10): 3576-80.

42. Barton RA, Harvey PH. Mosaic evolution of brain structure in mammals. Nature 2000; 405(6790): 1055-8.

43. Pollen AA, Nowakowski TJ, Chen J, et al. Molecular identity of human outer radial glia during cortical development. Cell 2015; 163(1): 55-67.

44. Stocker AM, O'Leary DD. Emx1 Is Required for Neocortical Area Patterning. PloS one 2016; 11(2): e 0149900.

45. Sherwood CC, Subiaul F, Zawidzki TW. A natural history of the human mind: tracing evolutionary changes in brain and cognition. J Anat 2008; 212(4): 426-54.

46. Borrell V, Gotz M. Role of radial glial cells in cerebral cortex folding. Current opinion in neurobiology 2014; 27: 39-46. 
47. Petryniak MA, Potter GB, Rowitch DH, Rubenstein JL. Dlx1 and Dlx2 control neuronal versus oligodendroglial cell fate acquisition in the developing forebrain. Neuron 2007; 55(3): 417-33.

48. Konno D, Iwashita M, Satoh Y, et al. The mammalian DM domain transcription factor Dmrta2 is required for early embryonic development of the cerebral cortex. PloS one 2012; 7(10): e46577.

49. Staes N, Sherwood CC, Wright K, et al. FOXP2 variation in great ape populations offers insight into the evolution of communication skills. Scientific reports 2017; 7(1): 16866.

50. Vargha-Khadem F, Gadian DG, Copp A, Mishkin M. FOXP2 and the neuroanatomy of speech and language. Nature reviews Neuroscience 2005; 6(2): 131-8. 51. Yang P, Wang Y, Macfarlan TS. The Role of KRAB-ZFPs in Transposable Element Repression and Mammalian Evolution. Trends Genet 2017; 33(11): 871-81. 52. Manolio TA, Collins FS, Cox NJ, et al. Finding the missing heritability of complex diseases. Nature 2009; 461(7265): 747-53.

53. Jacobs FM, Greenberg D, Nguyen N, et al. An evolutionary arms race between KRAB zinc-finger genes ZNF91/93 and SVA/L1 retrotransposons. Nature 2014; 516(7530): 242-5.

54. Ferguson-Smith AC. Genomic imprinting: the emergence of an epigenetic paradigm. Nat Rev Genet 2011; 12(8): 565-75.

55. Irie N, Weinberger L, Tang WW, et al. SOX17 is a critical specifier of human primordial germ cell fate. Cell 2015; 160(1-2): 253-68.

56. Kobayashi T, Zhang H, Tang WWC, et al. Principles of early human development and germ cell program from conserved model systems. Nature 2017; 546(7658): 416-20. 57. Grow EJ, Flynn RA, Chavez SL, et al. Intrinsic retroviral reactivation in human preimplantation embryos and pluripotent cells. Nature 2015; 522(7555): 221-5.

\section{Acknowledgements}

We thank members of the Surani lab for helpful discussions, as well as Drs Felipe Karam Teixeira, Thorold Theunissen, and Jose C. Silva for feedback on this work. SD received support through a core grant to the Wellcome Trust - Medical Research Council Stem Cell Institute, and through a grant to AS, MJK is an NIHR-funded Academic Clinical Lecturer, WWT receives support by a Croucher Foundation Fellowship and by the Newton Trust, EM from the Icelandic Research Fund (174564-053), PFC is a Wellcome Trust Principal Research Fellow (212219/Z/18/Z), and a UK NIHR Senior Investigator, who receives support from the Medical Research Council Mitochondrial Biology Unit (MC_UU_00015/9), the Medical Research Council (MRC) International Centre for Genomic Medicine in Neuromuscular Disease, the Evelyn 
Trust, and the National Institute for Health Research (NIHR) Biomedical Research Centre based at Cambridge University Hospitals NHS Foundation Trust and the University of Cambridge. The views expressed are those of the author(s) and not necessarily those of the NHS, the NIHR or the Department of Health. AS is a Wellcome Senior Investigator with a grant from the MRC. The Gurdon Institute receives a core grant from the Wellcome Trust and Cancer Research UK.

\section{Author contributions}

SD and AS conceived the project. SD designed and performed bioinformatics analyses. MJK, WWT and EM analysed the data and contributed to the discussions of the results, and TK and PFC provided essential resources. SD, MJK, and AS wrote the manuscript with input from all authors.

\section{Figure Legends}

Fig. 1: The potential of TE elements for transgenerational inheritance. $a, A$ schematic representing the stages of the human germline cycle. The inner circle indicates the average \% DNA methylation level of TE elements at those stages. The outer circle indicates the \% DNA methylation in somatic tissues. b, Pie chart and bar plot showing the percentage of elements, which intersect with 116,617 significantly hypermethylated regions in wk7-9 hPGCs for each TE subfamily indicated. TE elements were defined as those that cover at least $10 \%$ of the length of the hyper-methylated region (see also Supplementary Table 1). c, A schematic illustrating a hyper-methylated region ("escapee") within the intron of the SATB2 gene, which overlaps with multiple TE elements. The region's \% DNA methylation in wk9 hPGCs and gametes, and its conservation in non-human primates is shown. $\mathbf{d}$, A bar plot showing the percentage of TE elements in the human genome that are conserved in non-human primate and mammalian genomes (defined as TEs where at least $80 \%$ of the human TE element can be mapped to a syntenic region in the other species by liftOver). For each species, bars represent TE elements that are methylated in wk7-9 hPGCs (DNA methylation > 30\%) in red, and all annotated TE elements in blue (see also Supplementary Table 2). Whilst 75- 
$80 \%$ of non-methylated human TE elements are detected in apes and macaque, hPGCmethylated TE elements are more preferentially conserved in apes.

\section{Fig. 2: Association between hPGC-methylated regions and tissue-specific histone}

marks. a, Pie charts showing the percentage of human TE elements that intersect with established histone modification peaks in 7 fetal and 28 adult tissues (data from $\mathrm{NIH}$ Roadmap Epigenomics Project). The percentage of TE elements are shown for (top) those that are methylated in wk7-9 hPGCs (\% DNA methylation > 30\%) and (bottom) those that are non-methylated. $\mathbf{b}, \mathrm{A}$ heat map depicting the enrichment (Z-scores) for tissue-specific H3K9me3 ChIP-seq peaks (data from NIH Roadmap Epigenomics Project) in 9,902 human-specific hPGC-methylated TE elements, compared to 1,000 random sets. Shown are the human-specific TE elements that cannot be mapped to syntenic regions in non-human primates or other mammals, and with an enrichment Zscore of at least 10 for one cell type. c, Bar plots showing the percentage of TEs in each subfamily that intersect with $\mathrm{H} 3 \mathrm{~K} 9 \mathrm{me} 3$ and enhancer-related (H3K4me1+H3K27ac) ChIP-seq peaks in 7 fetal and 28 adult tissues (data from NIH Roadmap Epigenetics Project, see also Supplementary Table 4). TE elements are categorized as methylated (> $30 \%$ DNA methylation) or non-methylated in wk7-9 hPGCs. LINE L1 and SVA subfamilies are ranked according to their age. Log10-transformed $P$ values are indicated by bars. Younger primate-specific LINE L1 subfamilies (L1PA2-L1PA8) - but not the youngest (L1HS) - display the most significant differences in $\mathrm{H} 3 \mathrm{~K} 9 \mathrm{me} 3$ for methylated vs non-methylated TEs. Left: bar plot showing the fraction of TE elements for each subfamily that are methylated in wk7-9 hPGCs (DNA methylation > 30\%).

\section{Fig 3: Dynamic of epigenetic marks on hPGC-methylated TE elements during}

early human embryogenesis. a, A schematic showing the \% DNA methylation levels of in vitro hESCs and the most comparable human embryonic developmental stages. The analyses at these developmental stages characterize hPGC-methylated TE elements that lose H3K9me3 during the transition from naïve to primed hESCs. Some TE elements subsequently gain $\mathrm{H} 3 \mathrm{~K} 27 \mathrm{ac}$ and can act as enhancers at later developmental stages. b, A scatter plot showing for each TE subfamily the percentage of hPGCmethylated elements that intersect with enhancer-related (H3K4me1+H3K27ac) ChIPseq peaks in any of the 36 in vivo cell types (data from NIH Roadmap Epigenomics Project), compared to the average H3K9me3 log fold change (logFC) in primed vs naïve 
hESCs (data from Theunissen et al. 2016). The names of the TE subfamilies for which the DNA methylation and $\mathrm{H} 3 \mathrm{~K} 9 \mathrm{me} 3$ dynamics is shown in Fig $3 \mathrm{c}$ are printed in red. With the exception of SVAs, which gain H3K9me3 on average, most TE subfamilies lose H3K9me3 in primed hESCs. $6-12 \%$ of the TE elements of characteristic subfamilies, including LTR1D, HERV9 and HERVH, intersect with active enhancer marks. c, Scatter plots of the $\mathrm{H} 3 \mathrm{~K} 9 \mathrm{me} 3 \mathrm{logFC}$ compared to the difference in \% DNA methylation in primed vs naïve hESCs (data from Theunissen et al. 2016, Guo et al., 2017) for TE elements that are methylated (> $30 \%$ DNA methylation) or unmethylated in wk7-9 hPGCs. TEs that intersect with cell type-specific enhancers (H3K4me1+H3K27ac ChIP-seq peaks, data from NIH Roadmap Epigenomics Project) are shown in colour as indicated above the plots. Most TEs gain DNA methylation in primed hESCs while progressing from demethylated naïve hESCs. In contrast, hPGC-methylated TE elements lose H3K9me3 and become enhancers more frequently than unmethylated TEs, while non-methylated TEs gain H3K9me3 (see also Extended Data Fig. 3d).

Fig. 4: hPGC-methylated TE elements that switch to enhancers in the developing human brain. a, Scatter plot showing for each TE subfamily the percentage of elements that are DNA-methylated in wk7-9 hPGCs (y-axis) compared to the enrichment in 29,679 orthologous human and rhesus macaque enhancers (x-axis). Orthologous enhancers were defined by cross-referencing H3K27ac peaks determined at equivalent stages during corticogenesis (wk8.5 and wk12, data from Reilly et al., 2015, see Methods), with TE elements within the enhancers cross-referenced between human and rhesus macaque, respectively. b, Gene ontology (GO) term enrichment analysis for genes close to TEs that are differentially expressed between cortex and cerebellum $(\operatorname{abs}(\log 2 \mathrm{FC})>$ 2; adj $P$ value $<0.05$ ). TEs that were within $50 \mathrm{kB}$ distance upstream of the TSS or within the gene body of the genes were used for analysis. For each $\mathrm{GO}$ term red bars represent $\mathrm{BH}$-corrected $\mathrm{P}$ values for genes that are close to hPGC-methylated TEs, blue bars for genes that are close to non-methylated TE elements, and grey bars represent $P$ values for differentially expressed genes without considering TE information. c, Gene ontology (GO) term and Uniprot keyword enrichment analyses for genes that are within $50 \mathrm{kB}$ distance of hPGC-methylated and ape-specific LTR12C elements in active enhancers of human corticogenesis. d, Top co-expression module (module 5) that is enriched for ape-specific hPGC-methylated TE insertions. The genes representative of the GO terms found to be enriched for this module are labelled. Nodes are highlighted 
and scaled according to the number of TE insertions close to the gene during primate evolution. The module was partitioned into overlapping communities using the Louvain method (see Methods). e, Bar plots showing the evolutionary divergence of protein expression in human compared to chimp in the caudate nucleus $(\mathrm{CN})$ and the anterior cingulate cortex $(A C C)$ for two co-expression modules. The average expression of the genes in each module is shown for three replicates per species (data from Bauernfeind et al., 2015).

\section{Fig. 5: KRAB-ZFPs may influence the enhancer activity of hPGC-methylated TE}

elements in the brain. a, A heat map showing the average expression of KRAB zinc finger proteins (KZFPs) in wk7-9 hPGCs and in the developing brain. 29 KZFPs are differentially expressed between the cortex and cerebellum at any developmental stage, or between different stages of cortical development (CS22-wk17, 219 samples; log2FC > 1 ; $P$ value $<0.05)$. $\mathbf{b}$, Histograms showing the Pearson correlation coefficients between the expression of KZFPs and the expression of hPGC-methylated TEs of selected LTR and SVA subfamilies in the cortex and cerebellum (CS22-wk17, 219 samples). Selected enhancer-related TE subfamilies (such as LTR12C) are highlighted in red; and their expression is predominantly anti-correlated to KZFP expression. c, Representative genome browser tracks showing for TE elements, (bottom left) the KZFP and KAP1 ChIP-seq coverage, the \% DNA methylation in wk7-9 hPGCs, and the conservation in primate genomes. (top left) Scatterplots show the expression of the TE element versus the expression of the KZFP in CS22-wk17 cortex and cerebellar samples. TE expression and KZFP expression are anti-correlated. Boxplots show the average expression of the gene close to the TE element (bottom right) and the expression of the KZFP (top right) in CS22-wk17 cortex and cerebellar samples. d, Bar plots showing the gene-based binomial $P$ values for the association between selected brain-related and other/metabolic traits and TE subfamilies (see Methods). TE subfamilies, where $>40 \%$ of TE elements are hPGC-methylated and $>70 \%$ of TE elements are only detected in apes, are shown in red. See also Extended Data Fig. 6a and Supplementary Table 10. TE subfamilies are ranked by the number of genes that close to the GWAS SNPs. 


\section{Extended Data}

Fig. 1: Germline expression and age of hPGC-methylated TEs. a, A pie chart showing the intersection of hyper-methylated regions determined from two independent studies of DNA methylation in early hPGCs (see Methods, data from Tang et al., 2015 and Guo et al., 2015). b, A scatter plot showing for each TE subfamily the percentage of elements that are expressed in wk7-9 hPGCs (log2(normalized read counts) $>5$ ), and the percentage of elements that are methylated in wk7-9 hPGCs (DNA methylation > $30 \%$ ). Alu and LINE L1 elements are shown in a subfigure for clarity. Names are shown for TE families, of which at least $5 \%$ of annotated elements are methylated in wk7-9 hPGCs. c, A scatter plot showing for each TE subfamily the percentage of elements, which can be mapped to a syntenic region in chimp, gorilla, orangutan or gibbon genomes, and the percentage of elements that are methylated in wk7-9 hPGCs (DNA methylation $>30 \%$ ). Predominantly hominoid-specific TE subfamilies, which retain DNA methylation in hPGCs, are highlighted in blue.

Fig. 2: Association between hPGC-methylated regions and tissue-specific histone marks. a, A heat map showing the enrichment (Z-scores) for tissue-specific H3K9me3 ChIP-seq peaks (data from NIH Roadmap Epigenomics Project) in 9,902 human-specific hPGC-methylated TE elements that cannot be mapped to syntenic regions in nonhuman primates or other mammals, compared to 1,000 random sets. Only TE families with a Z-score of at least 10 for one cell type are shown. b, Related to Fig. 2c. Bar plots showing for each TE subfamily the percentage of elements that intersect with $\mathrm{H} 3 \mathrm{~K} 9 \mathrm{me} 3$ and enhancer-related (H3K4me1+H3K27ac) ChIP-seq peaks in 7 fetal and 28 adult tissues (data from NIH Roadmap Epigenomics Project) for TE elements that are methylated (> $30 \%$ DNA methylation) or unmethylated in wk7-9 hPGCs. TE subfamilies with significant differences in enhancer-related (H3K4me1+H3K27ac) peaks ( $P$ value < 1e-5, Pearson's Chi-squared test) for hPGC-methylated vs non-methylated regions are coloured in red. Left: bar plot showing the fraction of TE elements for each subfamily that are methylated in wk7-9 hPGCs (DNA methylation $>30 \%$ ). TE subfamilies are ranked according to the fraction of hPGC-methylated elements per TE subfamily.

Fig. 3: DNA methylation of hPGC-methylated TE elements in gametes. a, Bar plots showing for selected TE subfamilies the percentage of hPGC-methylated elements that 
are not methylated $(<20 \% 5 \mathrm{mC}$ ), partially methylated ( $>=20 \%$ and $<80 \%$ ), or methylated $(>=80 \%)$ in sperm, oocytes or blastocysts (data from Okae et al., 2014). TE subfamilies are ranked by the $5 \mathrm{mC}$ variability (coefficient of variation) in sperm (see also Supplementary Table 5). Older TE subfamilies are printed in bold. b, Density scatter plots of \% DNA methylation in sperm compared to oocytes, and in blastocysts compared to wk7 hPGCs for TE elements that are methylated (DNA methylation > 30\%) in wk7-9 hPGCs. c, Heatmaps showing the \% DNA methylation in wk7-9 hPGCs, oocyte, sperm and blastocysts (data from Okae et al., 2014) for TE elements that are methylated (DNA methylation $>30 \%$ ) or unmethylated in wk7-9 hPGCs. d, A scatter plot showing for each TE subfamily the average H3K9me3 logFC in primed vs naïve hESCs (data from Theunissen et al. 2016) for TEs that are methylated in hPGCs (x-axis) compared to TEs that are non-methylated (y-axis). For instance, for the LTR12C subfamily in the topleft quadrant, hPGC-methylated TE elements lose H3K9me3 on average, but nonmethylated TE elements of the LTR12C gain H3K9me3 in primed hESCs.

Fig. 4: Co-expression module analysis in the human fetal brain. a, A co-expression module analysis of 6,766 genes differentially expressed between cortex and cerebellum at the same developmental stage, or between different developmental stages of cortical development $(219$ samples, abs $(\log 2 F C)>1$, adj $P$ value $<0.05)$ using k-means clustering of the first 25 principal components (see Methods and Supplementary Table 7): (Top) A bar plot showing the number of genes in each of the 20 kmeans-derived modules. (Bottom) A PCA plot depicting the average PC scores of the genes in each coexpression module. Modules are coloured by their average log2FC between wk8-9 cortical and cerebellar samples with red modules being up-regulated in the cortex, and blue modules being up-regulated in the cerebellum. b, A bar plot showing the enrichment of hPGC-methylated TE elements close to the genes in each kmeansderived co-expression module (see Methods). TE elements are classified according to the primate lineage in which they first emerge. Modules are ranked by their enrichment of ape-specific TE elements. c, A principal component analysis (PCA) of TE expression during cortex, midbrain and cerebellar development (275 samples, CS14 to wk17). TE elements with at least $\log 2$ (normalized read counts) $>5$ were included in the analysis. Samples are coloured according to their eventual brain region in the adult brain and their developmental stages at the time of RNA extraction. hPGC wk5.5-wk7 samples are shown in black. d, Heat maps showing the expression of TE elements that are 
differentially expressed between cortex and cerebellum, or between different developmental stages of cortical development $(\operatorname{abs}(\log 2 \mathrm{FC})>2$, adj $P$ value $<0.05)$. In total, 9,363 TE elements were differentially expressed.

Fig. 5: Correlation between the expression of hominoid-specific KZFPs and TEs in the developing human brain. a, A schematic showing that KAP1 can be recruited to specific sites by KRAB zinc finger proteins. KAP1 acts as a scaffold for the assembly of a large repression complex consisting of the NuRD histone deacetylase complex and the H3 lysine 9-specific histone methyltransferase SETDB1. KAP1 further interacts with $\mathrm{HP} 1$, which in turn can bind to histone $\mathrm{H} 3$ that has been methylated on lysine 9 (H3K9me3). b, Ape-specific KZFPs that are expressed during human embryonic brain development. (Left panel) A heat map showing the enrichment (Z-scores) of KZFP ChIPexo peaks (data from Imbeault et al., 2017) in 9,902 human- and 52,654 ape-specific hPGC-methylated TE elements, compared to 1,000 random sets. Only those KZFPs and TE subfamilies that have a Z-score of at least 10 are shown. KZFPs that are differentially expressed between cerebellum and cortex at any developmental stage, or between different stages of cortical development are shown in blue (see also Fig. 5a). (Right panel) \% sequence identity of the human KZFP gene to its one-to-one ortholog in nonhuman primates. c, Bar plots showing the percentage of TEs in each subfamily that intersect with KZFP ChIP-exo peaks (data from Imbeault et al., 2017). TE elements are categorized as methylated (DNA methylation > 30\%) or non-methylated in wk7-9 hPGCs. KZFP binding is more prevalent for hPGC-methylated in comparison to nonmethylated elements of the same TE subfamily ( $P$ value $<0.01$, Pearson's Chi-squared test), indicating that KZFPs might regulate the protection of hPGC-methylated TE elements from DNA de-methylation. Left: bar plot showing the fraction of TE elements for each subfamily that are methylated in wk7-9 hPGCs. d, Bar plots showing the percentage of TEs that have a significant correlation in expression (Pearson correlation coefficient $>0.5$, $\mathrm{P}$ value $<0.001$ ) with their neighbouring genes (distance $<15 \mathrm{kB}$ ) for hPGC-methylated and non-methylated TEs. TE elements are further categorized into three classes: (1) those intersecting with H3K9me3 ChIP-seq peaks, (2) those intersecting with enhancer-related $(\mathrm{H} 3 \mathrm{~K} 4 \mathrm{me} 1+\mathrm{H} 3 \mathrm{~K} 27 \mathrm{ac}) \mathrm{ChIP-seq} \mathrm{peaks,} \mathrm{and} \mathrm{(3)} \mathrm{those}$ intersecting with enhancer-related ChIP-seq peaks and with at least two KZFP peaks (data from NIH Roadmap Epigenomics Project, and from Imbeault et al., 2017). 
Fig. 6: Association of hPGC-methylated TEs with human traits and diseases. a, Bar plots showing for selected hominoid-specific TE subfamilies the gene-based binomial $P$ values for the association with the top 10 most significantly associated GWAS traits. GWAS traits are ranked by the number of genes shared with the TE subfamily. 


\section{Methods}

\section{DNA methylation analyses}

Description of data sets. DNA methylation data sets were obtained from public sequence archives for week 10-11 hPGCs (Guo et al., 2015; GEO: Accession: GSE63818), for gametes (oocyte and sperm) and blastocysts (Okae et al., 2014; Japanese Genotype-phenotype Archive: Accession: JGAS00000000006), and naïve and primed hESCs (Guo et al., 2017; GEO Accession: GSE90168). Our previously published data set from wk7-9 hPGCs (Tang et al., 2015) and data set from wk10-11 hPGCs from Guo et al. (2015) both represent the most de-methylated stage in primordial germ cells. Naïve hESCs most closely resemble the blastocyst stage, and both data sets represent a further wave of DNA de-methylation in the pre-implantation embryo.

Data processing. PBAT reads were pre-processed by removing $4 \mathrm{nt}$ from the 5 ' end of the first read, and $15 \mathrm{nt}$ from the second read to remove random primers using Trim Galore! (parameters: '--paired --clip_R1 4 --clip_R2 15 -three_prime_clip_R1 1 three_prime_clip_R2 1 -q 30'). Quality-trimmed reads were aligned to the human reference genome (GRCh37/hg19 and GRCh38/hg38) using Bismark (parameters: '-n 2 -I 100 --bowtie1 -pbat'). Duplicates were removed using Bismark deduplicate. Alignments were further processed with MethPipe. Bam files were converted by to-mr, and methylation levels on $\mathrm{CpGs}$ were determined with methcounts, and symmetric CpG sites on opposite strand were merged by symmetric-cpgs. UCSC genome browser tracks (bigwig files) were generated by calculating a smoothed average of the DNA methylation levels of $\mathrm{CpGs}$ on 100nt-sliding windows (step $10 \mathrm{nt}$ ) using custom Perl scripts. Methylation levels of the hypo-methylated hPGC genomes were inverted (1- \% methylation), and hyper-methylated regions were determined using the Methpipe $\mathrm{hmr}$ command. Hyper-methylated regions obtained from individual and pooled replicates were merged, and only hyper-methylated with at least $30 \%$ methylation in one individual or pooled replicate were retained. Published DNA methylation data sets were processed in the same way, except that end repair was not performed on WGBS-seq reads, and they were aligned with the '-directional' option.

\section{ChiP-seq analyses}

ChiP-seq data sets for KAP1 and H3K9me3 were obtained from the NCBI Gene Expression Omnibus (GEO) for human naïve and primed hESCs (Theunissen et al., 
2015; Accession: GSE75868), and ChIP-exo data sets for 238 KZNFs in HEK 293T cells (Imbault et al., 2017; Accession: GSE78099). Reads were pre-processed with Trim Galore! (parameters: '--length 25 -q 30'), and aligned to the human reference genome (GRCh37/hg19 and GRCh38/hg38) using bowtie (parameters: '-M 1 -v2 -best --strata' to filter unique alignments only. Narrow peaks were called with macs2 callpeaks (parameters: '-g 3e9 -q 0.05').

\section{Analyses of transposable elements}

DNA methylation. RepeatMasker-annotated TE elements were obtained for the GRCh37/hg19 and GRCh38/hg38 human genomes from the UCSC Genome Table Browser. \% DNA methylation levels on transposable elements were determined by using MethPipe roimethstats considering only CpGs with at least $5 \mathrm{X}$ coverage. hPGCmethylated TE elements were required to have at least $30 \%$ methylation in one individual or pooled replicate, and to intersect with a hyper-methylated region by $50 \%$ of the length of the TE element.

Mapping of TE elements to non-human primate genomes. Pairwise alignments files with non-human primate genomes (and other mammals) were obtained from UCSC Genome Browser Downloads. Human RepeatMasker-annotated TE elements were mapped to the genomes of other species using liftover. TE elements which mapped by 0.9-1.1 of their length in human to the other species were considered to be conserved.

TE expression analysis in hPGCs and brain. RNA-seq reads were preprocessed using Trim Galore!, and reads from hPGC and brain RNA-seq data sets were trimmed to uniform length $(50 \mathrm{nt})$ to be comparable. Reads were aligned to the human reference genome (GRCh37/hg19 and GRCh38/hg38) using bowtie (parameters: "-m 1 -v 2 -best -strata") to filter the best and unique alignments only, in order to identify precise locations of TE expression. Read counts on TE elements were obtained by featureCounts, and differential expression analyses were performed using the $\mathrm{R}$ Bioconductor DESeq package. A principal component analysis for TEs with an expression of at least log2 (normalized counts) $>2$ was performed using the $\mathrm{R}$ prcomp function (see below for further details on the brain RNA-seq data set). 
Intersection with tissue-specific histone marks. Narrow peaks for 36 in vivo cell types, and for H1/H9 hESCs were obtained from the NIH Roadmap Epigenomics Project. The in vivo cell types were selected, for which enhancer-related histone marks (H3K4me3+H3K27ac), H3K9me3 and H3K36me3 were all available. For the H3K9me3 enrichment analysis in Fig. $2 b, 1,000$ random peak sets of the same size as the peaks called for the Roadmap Epigenomics data sets and within the vicinity of genes $(<50 \mathrm{kB})$ were generated with bedtools shuffle command. $\mathrm{P}$ values and $\mathrm{Z}$ scores for the enrichment of $\mathrm{H} 3 \mathrm{~K} 9 \mathrm{me} 3$ were estimated by counting the overlap $N$ with the 1,000 randomly generated peak sets, where the $P$ value is defined as $(N+1) /(1000+1)$. Pearson chi-squared tests in Fig. 2a (global) and 2c (TE subfamily-based), of observed proportions of TEs that intersect with histone marks for methylated vs non-methylated TEs were performed with the $R$ chisq.test function.

Enrichment of hPGC-methylated TE elements in brain enhancers. ChIP-seq data sets for enhancer-related histone marks (H3K4me2 and H3K27ac) from the whole cortex at wk8.5 and primitive frontal and occipital tissues at wk12 for human and rhesus macaque were obtained from GEO (Reilly et al., 2015; Accession: GSE63649), preprocessed with the standard ChIP-seq protocol (see above), and aligned to the human (GRCh37/hg19) and rhesus macaque (rheMac8) reference genomes. Narrow peaks were called with macs2 callpeaks. Active enhancers in human and rhesus macaque were defined as $\mathrm{H} 3 \mathrm{~K} 27 \mathrm{ac}$ peaks that intersected with an $\mathrm{H} 3 \mathrm{~K} 4 \mathrm{me} 2$ peak, and did not intersect with an annotated promoter region. Human enhancer-related $\mathrm{H} 3 \mathrm{~K} 27 \mathrm{ac}+\mathrm{H} 3 \mathrm{~K} 4 \mathrm{me} 2$ peaks were then mapped to the rhesus macaque genome by liftover, and human enhancers that intersected with an $\mathrm{H} 3 \mathrm{~K} 27 \mathrm{ac}+\mathrm{H} 3 \mathrm{~K} 4 \mathrm{me} 2$ peak in rhesus macaque by at least $30 \%$ were considered conserved. We then cross-referenced and identified the transposable elements in the human enhancer regions that were not present in the orthologous region in macaque, and their DNA methylation in wk7-9 hPGCs, in order to identify hPGC-methylated TEs that were selected or newly inserted during human evolution and gain enhancer activity at this stage in human cortical development.

\section{Brain RNA-seq data analysis}

Description of data sets. We analysed in total 275 cortex, midbrain, and cerebellum RNA-seq samples from the developing human brain by access to the HDBR resource ${ }^{40}$. 
The brain RNA-seq data set is unique in its age range (CS14-wk17), and number of brains (172) studied, in particular the samples earlier than wk8 of brain development. The majority of samples are from the time between wk9-12, where the major brain regions are established and the early stages of cortex development occur.

Data processing. Paired-end brain RNA-seq reads from the HDBR resource were trimmed of adapters using Trim-Galore!. Pre-processed reads were aligned to the human reference genome using TopHat2. Read counts on ENSEMBL protein coding, lincRNA and processed transcripts were obtained by featureCounts. Normalization and differential expression analyses were performed using the $\mathrm{R}$ Bioconductor DESeq2 package.

Gene co-expression modules in brain. We identified 6,766 genes differentially expressed between cerebellum and cortex, or between different stages of cortical development (CS14-wk17, 219 samples, normalized expression > 5 in one condition; $\operatorname{abs}(\log 2 F C)>1$, adj $P$ value $<0.05)$. First, we reduced the dimensionality of the gene expression matrix (genes with log2 normalized expression $>5$ in at least 2 samples were selected) by performing a Principal Component Analysis (PCA) using the R prcomp function on the center-scaled gene expression values. We then performed a k-means clustering on the first 25 principal components of the 6,766 differentially expressed genes using the $\mathrm{R}$ kmeans function to define 20 gene co-expression modules. We calculated the average expression log2FC between wk8-9 cortex and cerebellum for the genes in each $\mathrm{k}$ means-derived module, since KZFPs show a distinct expression in the cortex at this developmental stage, and visualized the average log2FC of each module in PC2 vs PC1 space in Fig. 4a. Visualization of the adjacency matrix of a module (where nodes represent genes, and edges represent the Pearson correlation coefficient of gene expression between two genes in all cortex and cerebellar samples) in Fig. $4 d$ were performed with the $\mathrm{R}$ igraph package. In addition, we performed the Louvain community detection method on each module using the cluster_louvain function of the $R$ igraph package.

For each gene we determined the TE elements within $50 \mathrm{kB}$ upstream of the transcriptional start site (TSS) or within the gene body. TE elements were classified according to their age depending on the non-human primate genome (human only, great 
apes or apes), where they were still conserved. The enrichment score for hPGCmethylated TE elements within gene co-expression modules according to their age in Extended Data Fig. 4b was determined as log2 (TE count by age / \# of genes in module)/(TE count / \# of all expressed genes).

\section{Analyses of KRAB zinc-finger proteins (KZFPs)}

614 human genes with KRAB box (Pfam: PF01352) and C2H2-type zinc finger domains (Pfam: PF00096) and their gene orthology information for non-human primates, and mouse were obtained from ENSEMBL (version 96). Since hPGC-methylated TEs were predominantly hominoid-specific, we determined in Extended data Fig. 5b all KZFPs that have newly emerged or significantly evolved their gene structure in apes. We compared their mutual \% sequence identity versus all non-human primate genomes and mouse. KZFPs with transcripts that share a mutual $\%$ seq id $>90 \%$ with any macacque transcript (including "many-to-many" orthologs) were excluded. We further required that KZFPs were differentially expressed between cerebellum and cortex, or between different stages of cortical development (CS14-wk17, 219 samples, normalized expression $>5$ in one condition; abs $(\log 2 F C)>1$, adj $P$ value $<0.05)$. For KZFP binding enrichment analysis in hominoid-specific TE subfamilies, 1,000 random regions of the same size as the TE elements were generated with bedtools shuffle command. $\mathrm{P}$ values and $Z$ scores for the enrichment of KZFP peaks were estimated by counting the overlap $N$ with the 1,000 randomly generated TEs, where the $P$ value is defined as $(\mathrm{N}+1) /(1000+1)$. $\mathrm{P}$ values for the Pearson correlation coefficient between KZFP and TE expression in Fig. 5b were determined with the R cor.test function.

\section{Association of hPGC-methylated TEs and GWAS traits}

The most recent GWAS catalog was obtained from EBI (https://www.ebi.ac.uk/gwas/docs/downloads, Jan 2019); additional data on loci influencing human intelligence were obtained from Sniekers at al., 2017. We considered only high-confidence GWAS SNPs with an adjusted $P$ value $<1 e-10$. Direct overlap of hPGC-methylated TEs or hyper-methylated regions with SNPs were rare; we therefore identified the nearest genes of SNPs and hPGC-methylated TEs within $50 \mathrm{kB}$ of their transcriptional start sites or within the gene body, and evaluated the statistical significance of the overlap between gene sets associated with GWAS traits and those associated with hPGC-methylated TEs for each TE subfamily using a binomial test. P 
values were determined by using the $\mathrm{R}$ pbinom function and adjusted for multiple testing using the Benjamini-Hochberg $(\mathrm{BH})$ correction. 
Figure 1bioRxiv preprint doi: https://doi.org/10.1101/2020.03.19.998930; this version posted March 20, 2020. The copyright holder for this preprint (which was not certified by peer review) is the author/funder, who has granted bioRxiv a license to display the preprint in perpetuity. It is a made available under aCC-BY-NC-ND 4.0 International license.

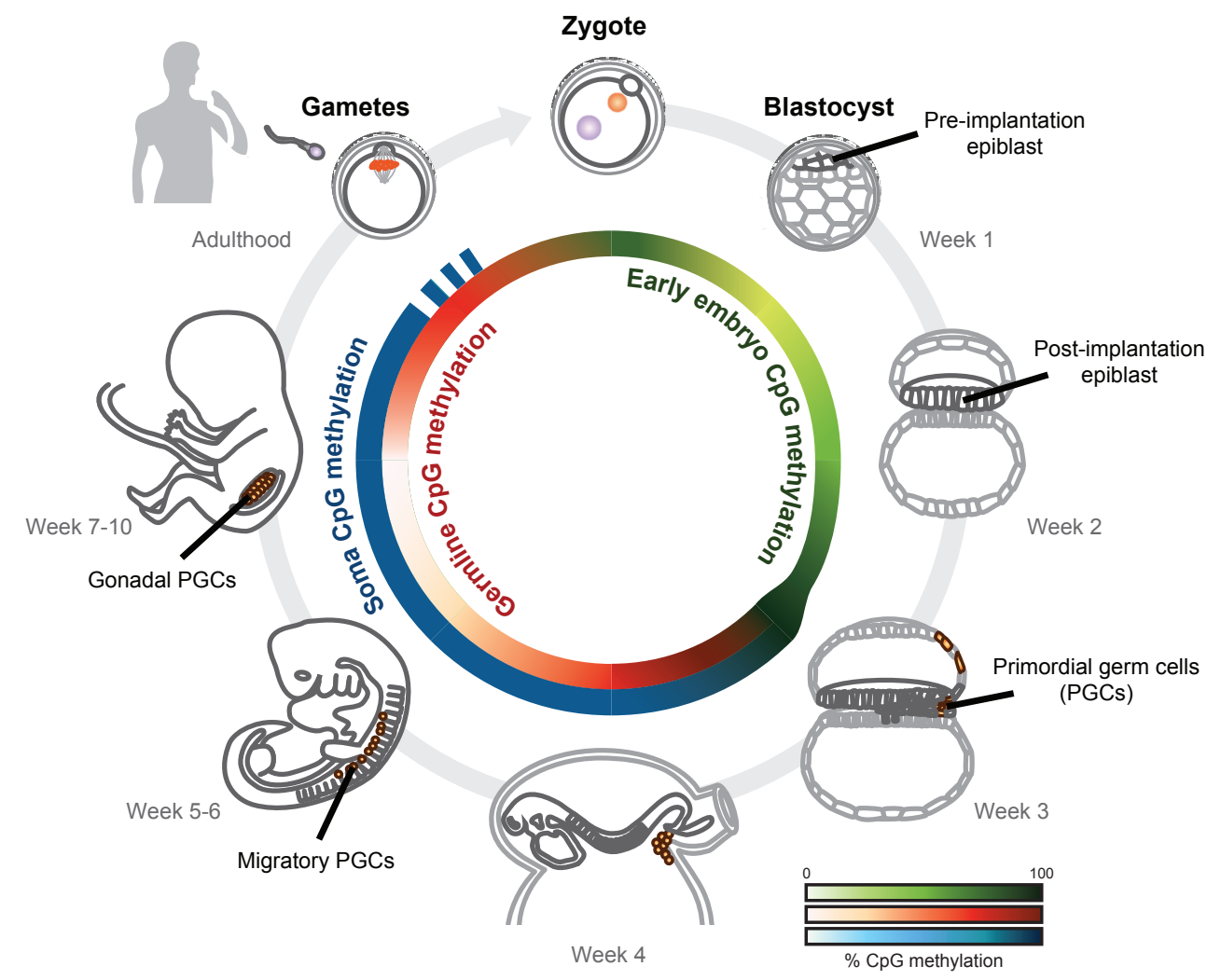

b

wk7-9 hPGC hyper-methylated regions $(n=116,617)$

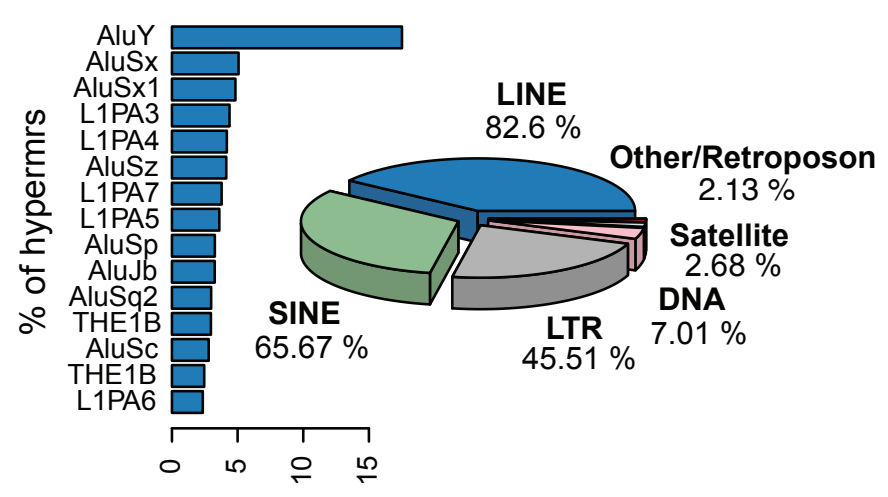

C

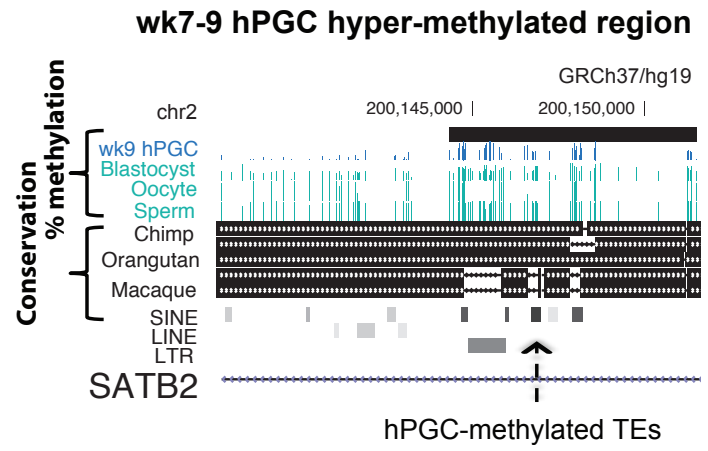

d

hPGC-methylated TEs $(n=117,161)$
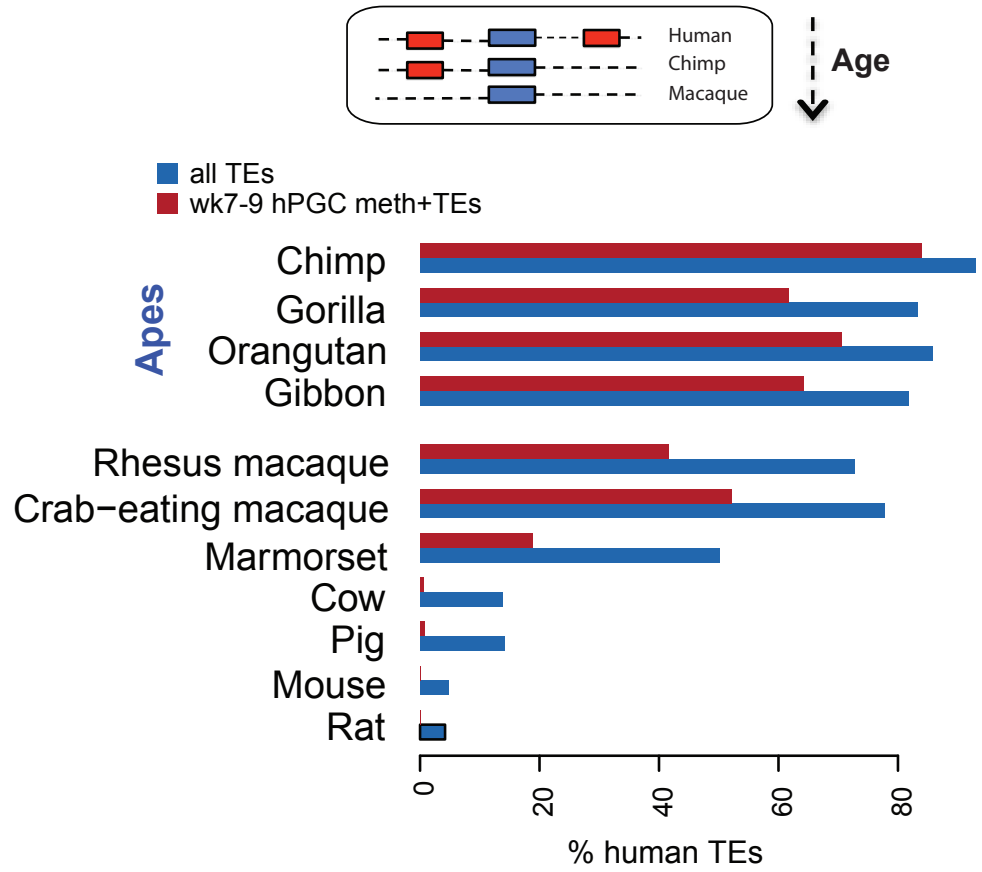
Figure BioRxiv preprint doi: https://doi.org/10.1101/2020.03.19.998930; this version posted March 20, 2020. The copyright holder for this preprint (which was not certified by peer review) is the author/funder, who has granted bioRxiv a license to display the preprint in perpetuity. It is

a

\section{hPGC meth+}

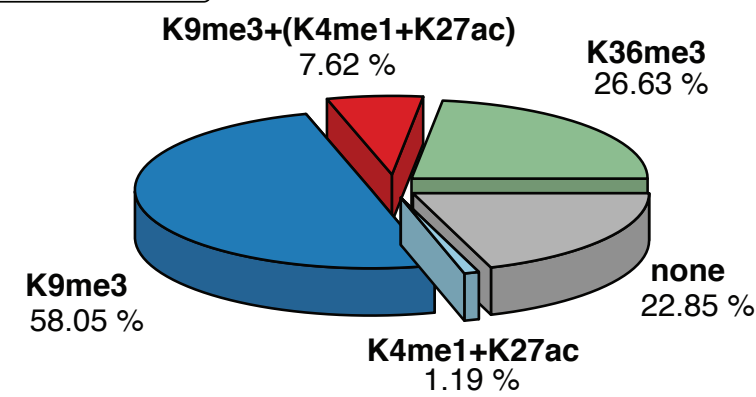

\section{hPGC meth-}

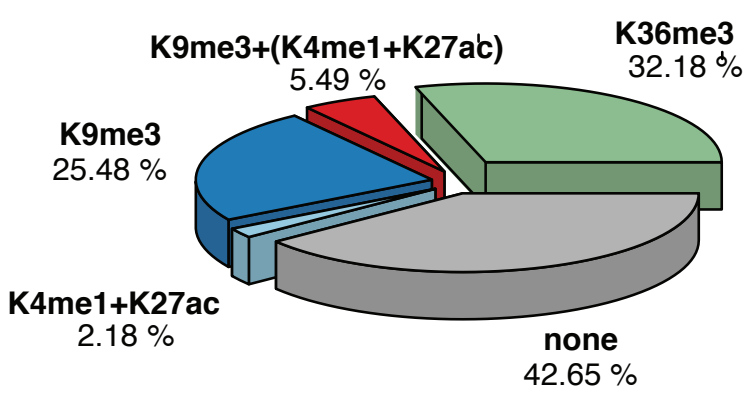

C
Tissue-specific $\mathrm{H} 3 \mathrm{~K} 9 \mathrm{me} 3$ in 9,902 human-specific hPGC-methylated TE elements

Enrichment vs 1,000 random sets
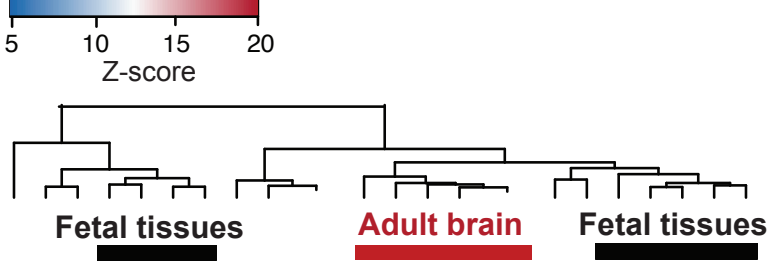

$1 \mathrm{HS}$

L1PA2

L1PA3

L1PA4

LPA5

$1 \mathrm{PA6}$

L1P1

L1PA7

L1PB1
K9me3

K9me3
K4me1+K27ac
K9me3+(K4me1+K27ac)
none

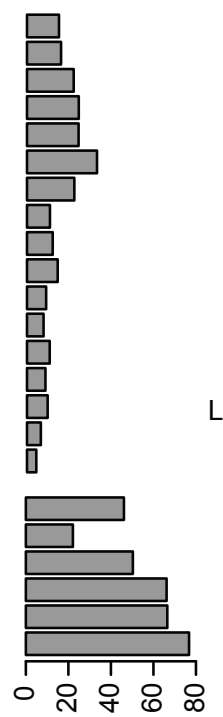

$\%$ TEs methylated in wk7-9 hPGCs

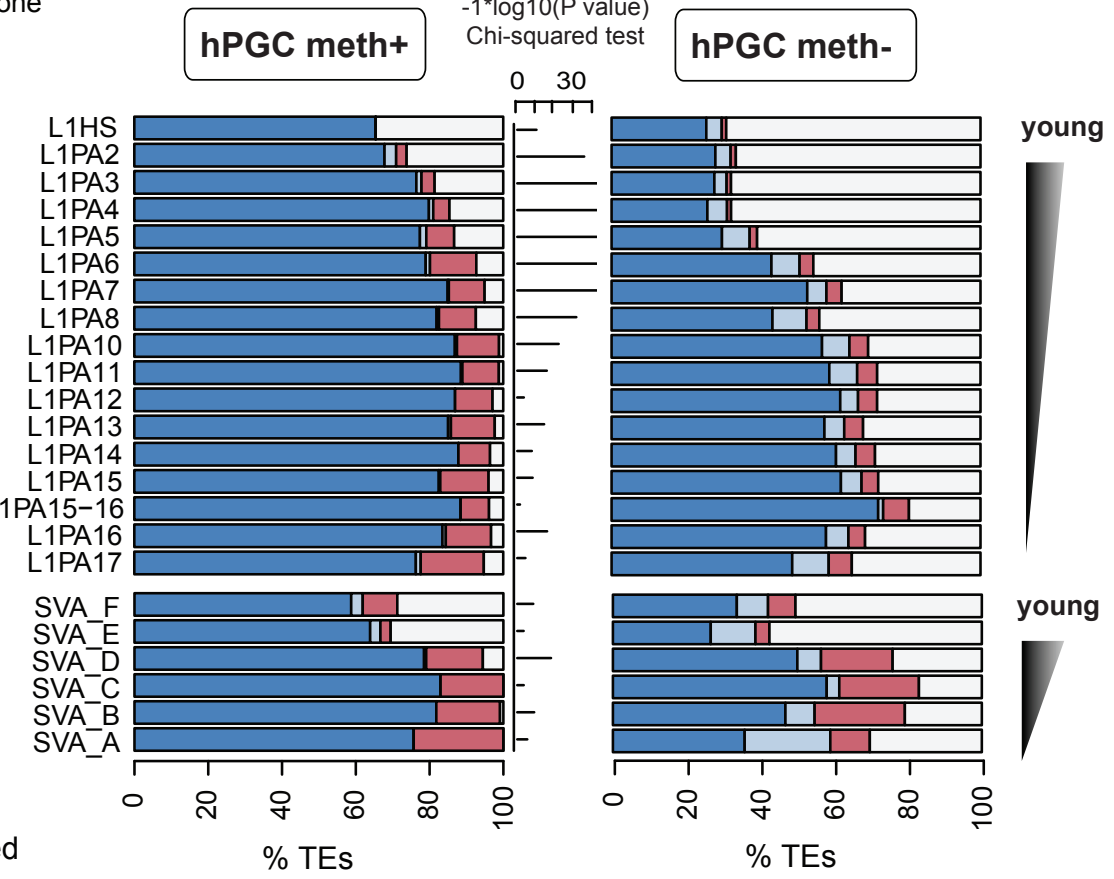


Figure 3bioRxiv preprint doi: https://doi.org/10.1101/2020.03.19.998930; this version posted March 20, 2020. The copyright holder for this preprint (which was not certified by peer review) is the author/funder, who has granted bioRxiv a license to display the preprint in perpetuity. It is

a

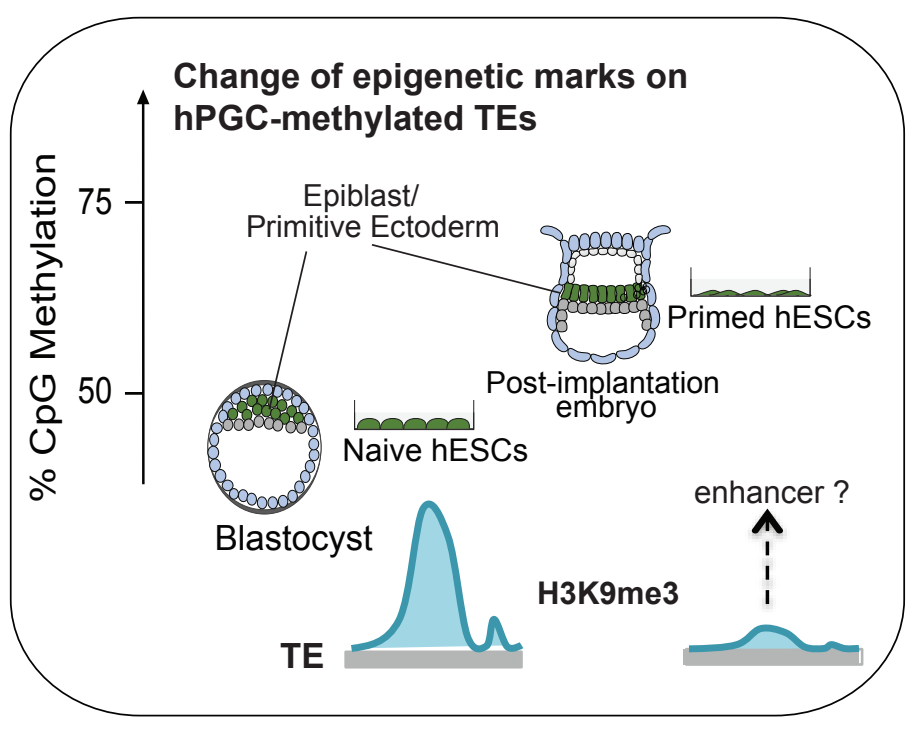

C

\section{hPGC meth+ TEs}

that intersect enhancers $(\mathrm{K} 4 \mathrm{me} 1+\mathrm{K} 27 \mathrm{ac})$ in:

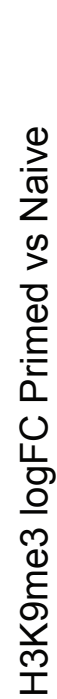

- H1/H9 ESC

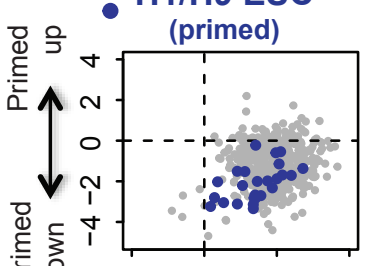

- Brain
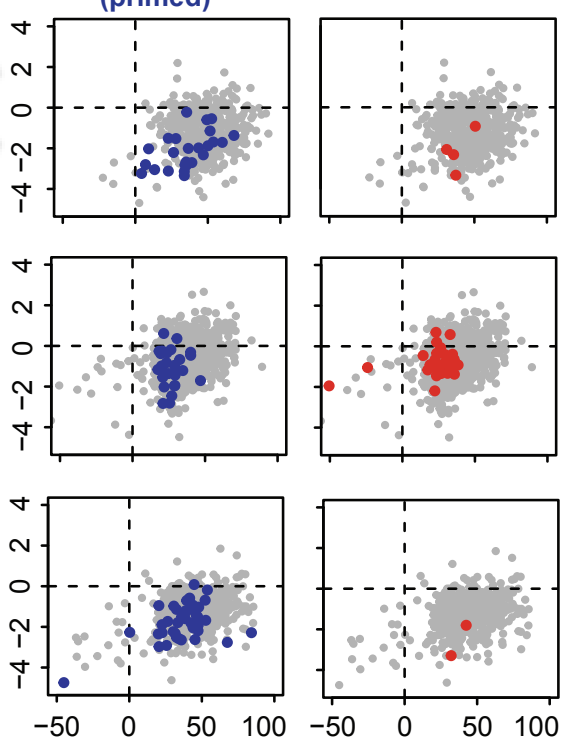
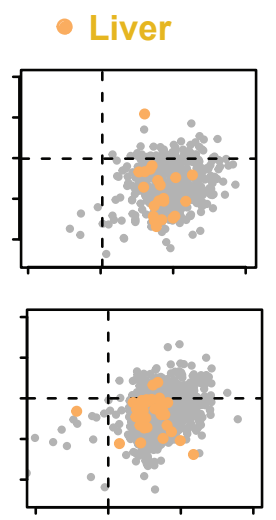

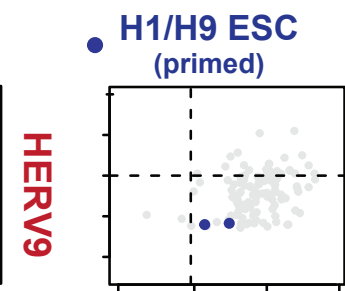

- Brain

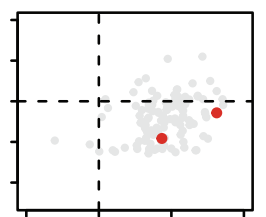

- Liver
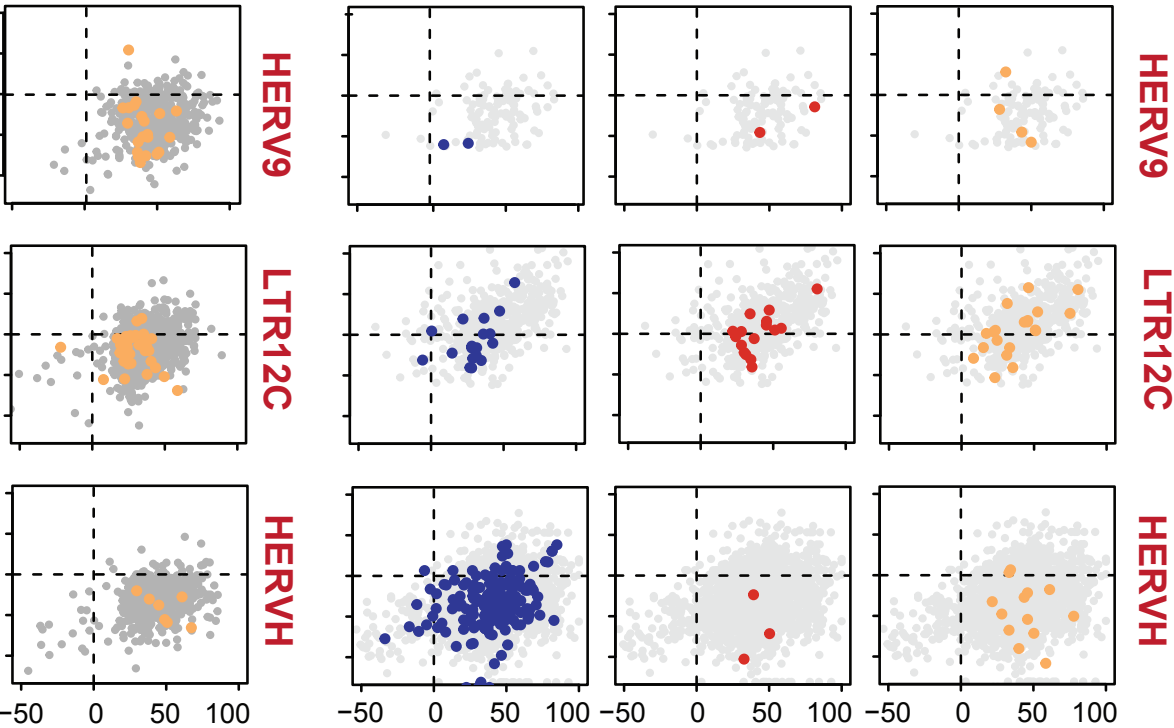

검

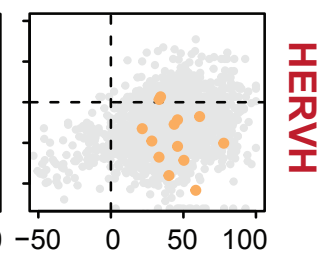

Difference \% DNA methylation Primed vs Naive hESCs 
Figure 4bioRxiv preprint doi: https://doi.org/10.1101/2020.03.19.998930; this version posted March 20, 2020. The copyright holder for this preprint (which was not certified by peer review) is the author/funder, who has granted bioRxiv a license to display the preprint in perpetuity. It is made available under aCC-B $\mathbf{b}^{-N C-N D} 4.0$ International license.

\section{8,551 orthologous enhancers}

(human and rhesus macaque, H3K27ac+

wk8.5 and w12 cortex, Reilly et al. 2015)

- hominoid-specific insertions
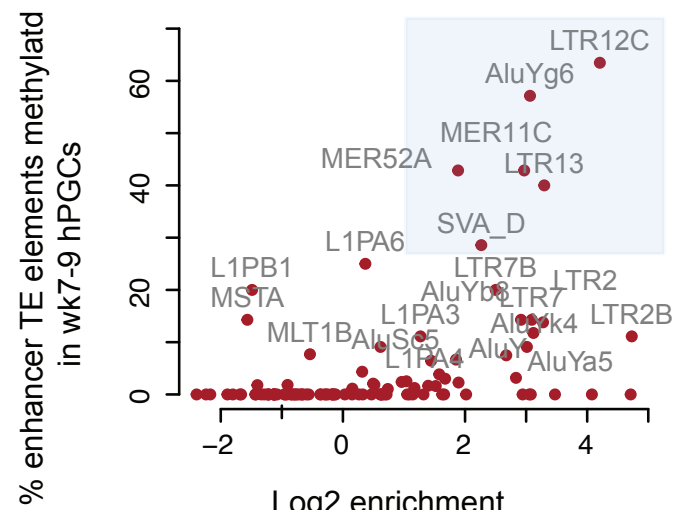

C

\section{Gene Ontology (GO)}

- Differentially expressed genes

$(\operatorname{abs}(\log 2 \mathrm{FC})>4 ; 503)$

Genes downstream of differentially expressed TEs: - hPGC meth+ (\% DNA methylation > 30\%) (155)

- hPGC meth- (455)

\section{LTR12C}

LTR12C
negative regulation of cell adhesion
neurogenesis
immunoglobulin domain
negative regulation of astrocyte differentiation
negative regulation of astrocyte differentiation

d neural crest cell migration adult behavior cell fate commitment glutathione metabolic process vocalization behavior neurotransmitter secretion regulation of MAPK cascade organ morphogenesis male gonad development axonogenesis neuron migration central nervous system development phosphatidylinositol signaling social behavior

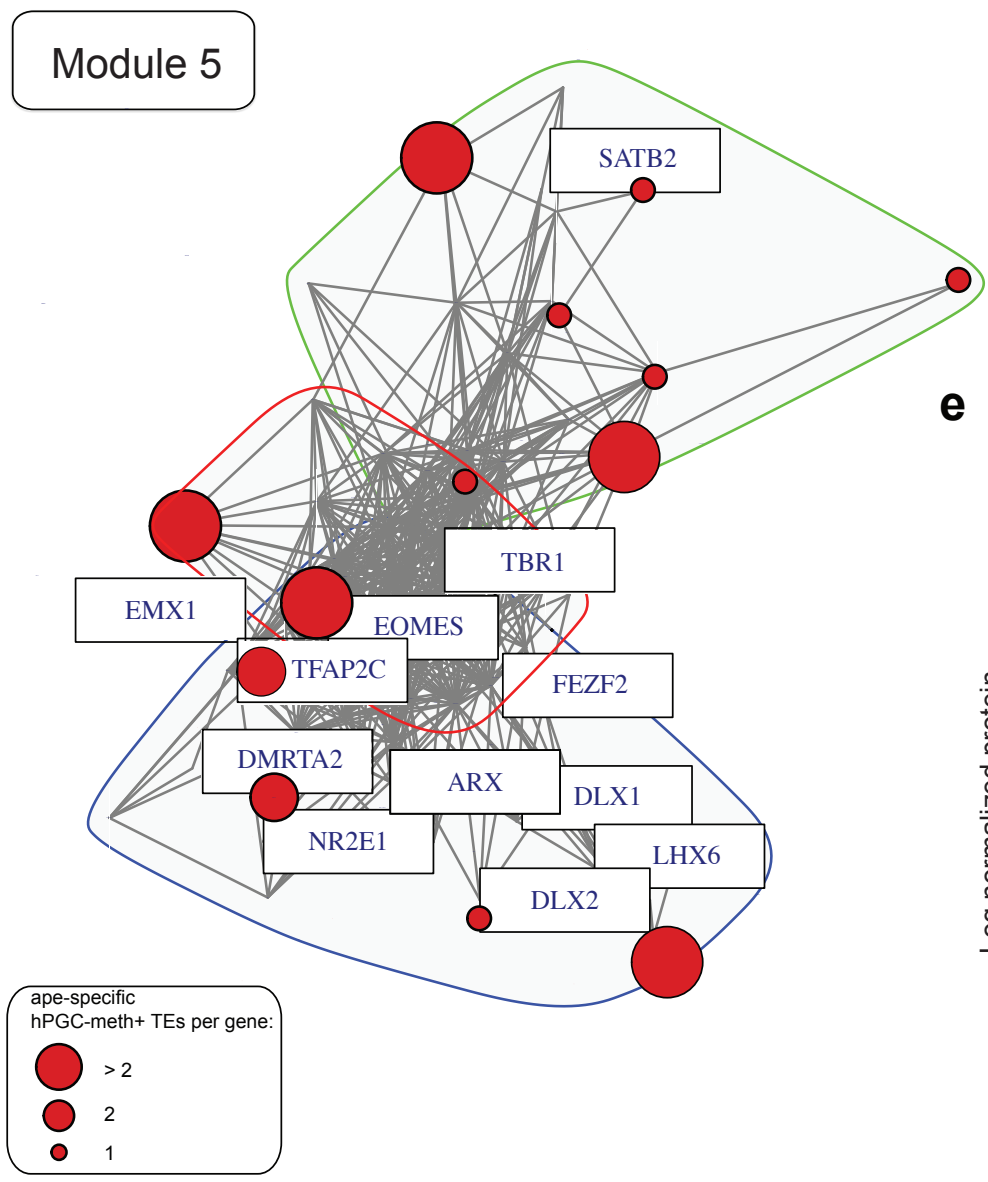

CN: caudate nucleous

ACC: anterior cingulated cortex

$$
\begin{gathered}
\text { Module } 5 \\
\text { (cortex-specific) } \\
\text { CN }
\end{gathered}
$$

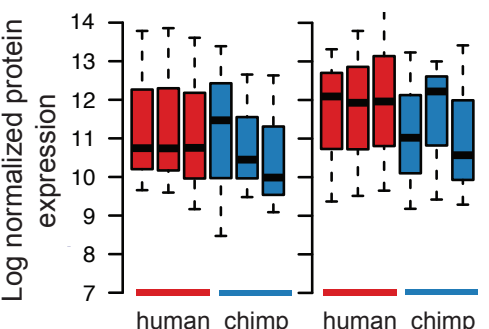

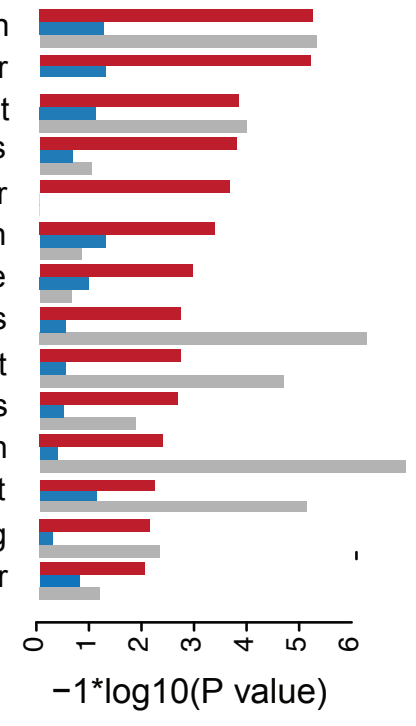

$-1{ }^{*} \log 10(\mathrm{P}$ value $)$

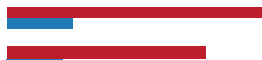

r

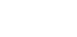

Module 9

(cerebellum-specific)

CN

ACC

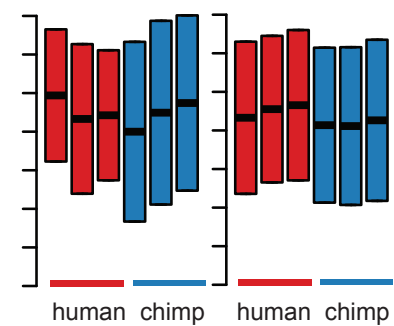


Figure 5 5 ioRxiv preprint doi: https://doi.org/10.1101/2020.03.19.998930; this version posted March 20, 2020. The copyright holder for this preprint (which was not certified by peer review) is the author/funder, who has granted bioRxiv a license to display the preprint in perpetuity. It is made available under a C $^{-B Y}$-NC-ND 4.0 International license.

KZFPs with dynamic expression in brain

\begin{tabular}{|c|c|}
\hline & Apes \\
\hline $\begin{array}{l}02468 \\
2 \text { 2(norm counts) }\end{array}$ & $\begin{array}{l}\text { - Old world monkeys } \\
\text { - Eutherians }\end{array}$ \\
\hline
\end{tabular}
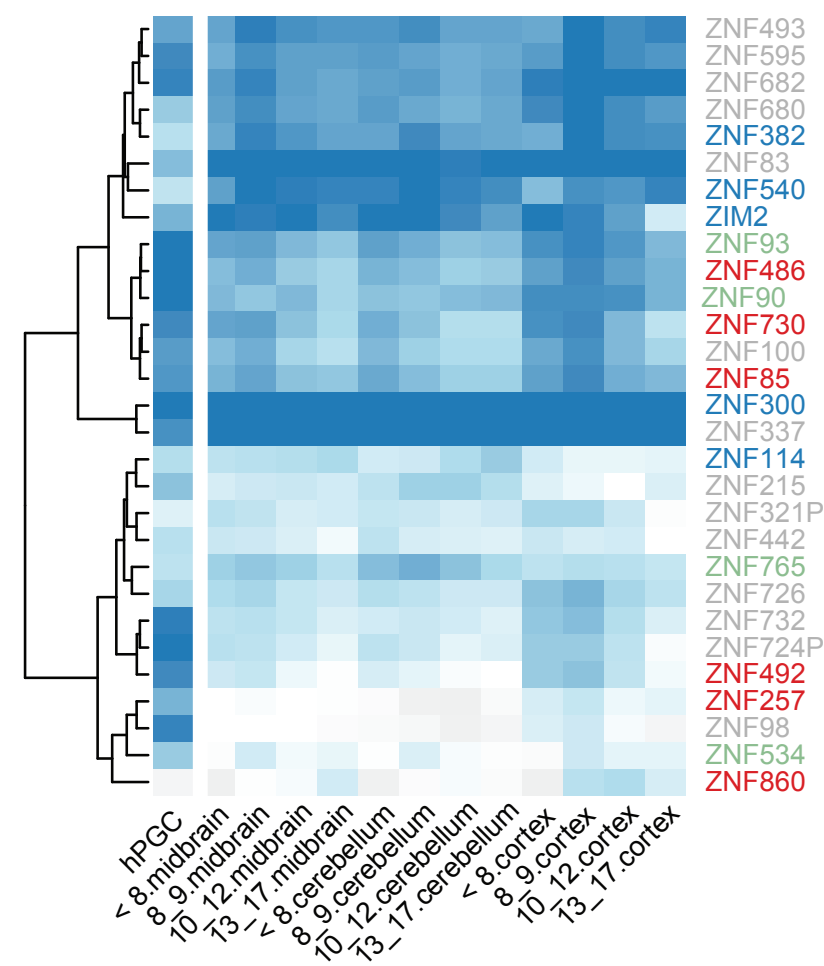

b

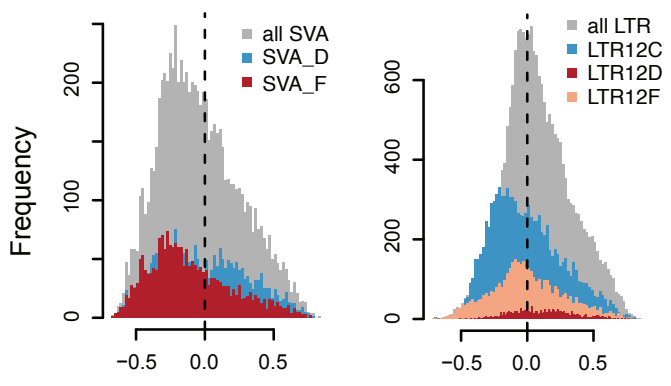

KZFP-TE expression correlation in brain

d

Intelligence

(Sniekers et al., 2017)
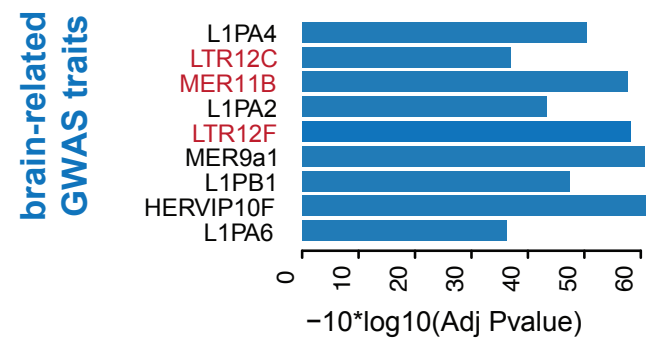

\section{Body height}

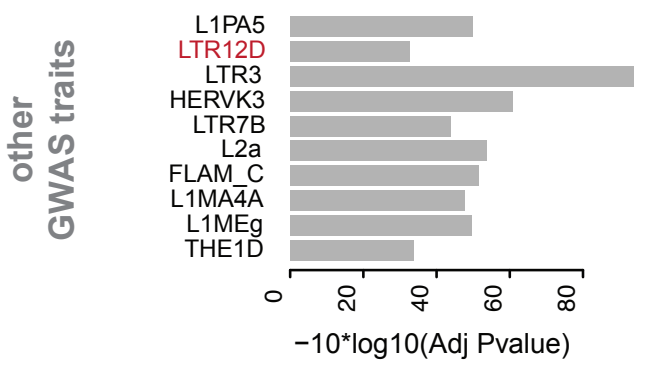

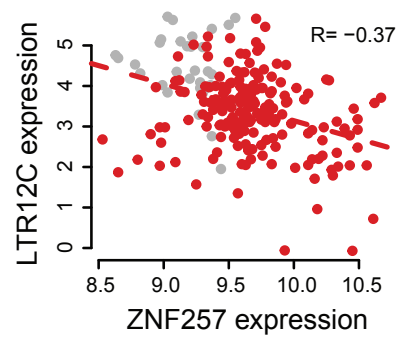
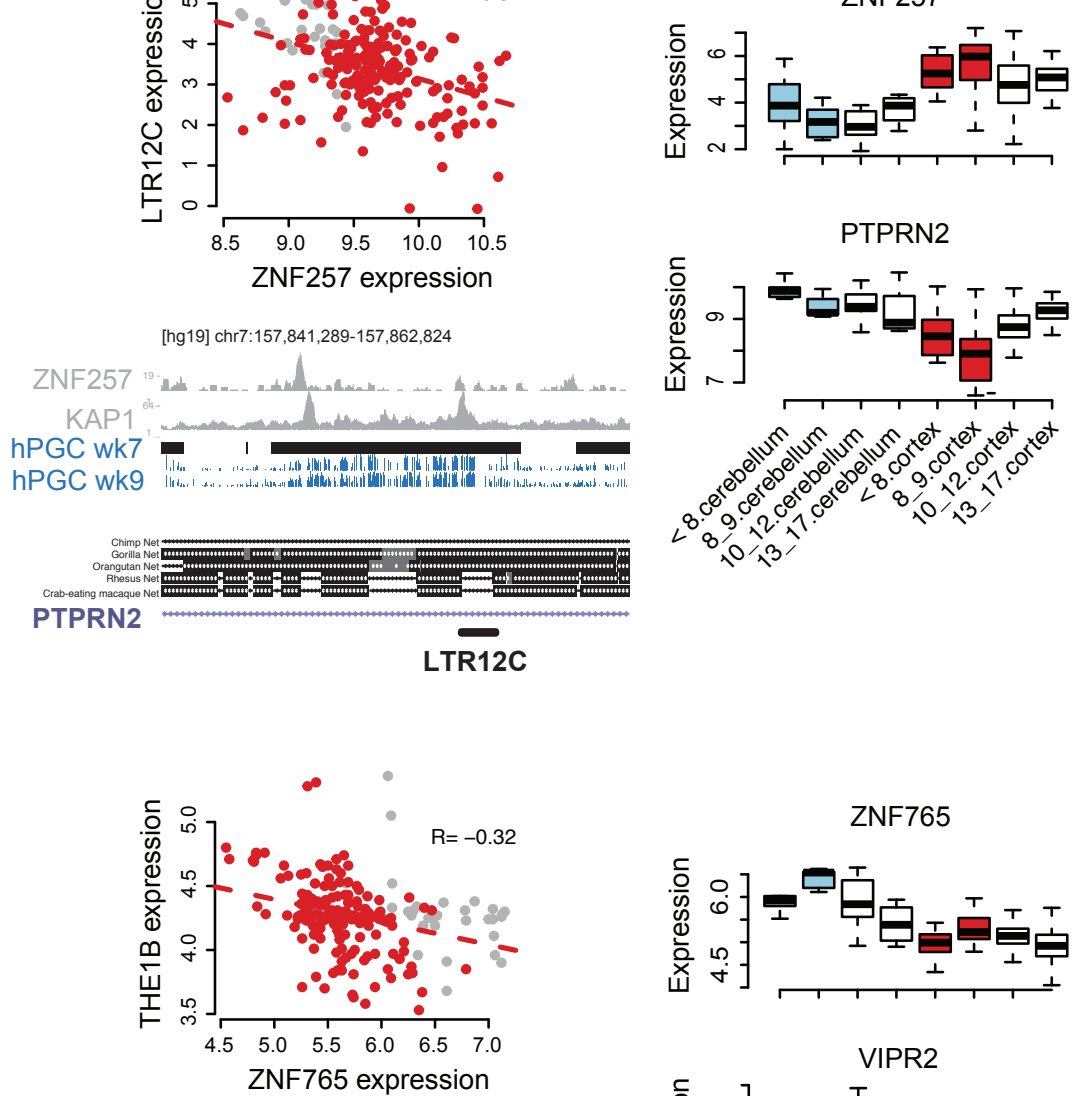

[hg19] chr7:158,891,194-158,897,876

ZNF765

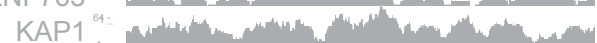
hPGC wk7 hPGC wk9

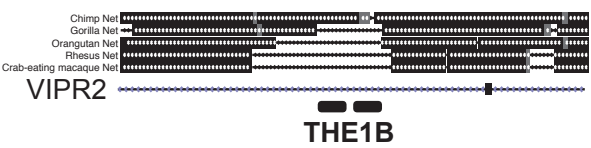

THE1B
Alzheimer's disease

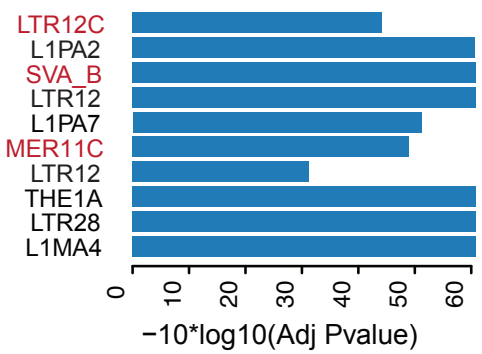

Type II Diabetes mellitus

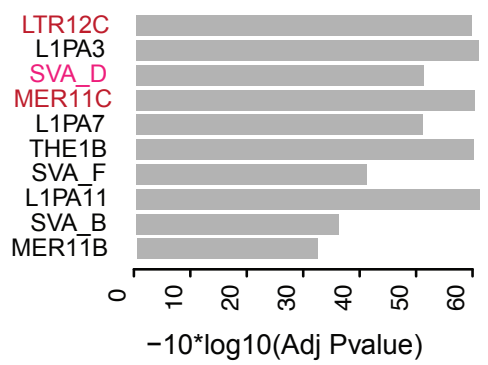

Schizophrenia

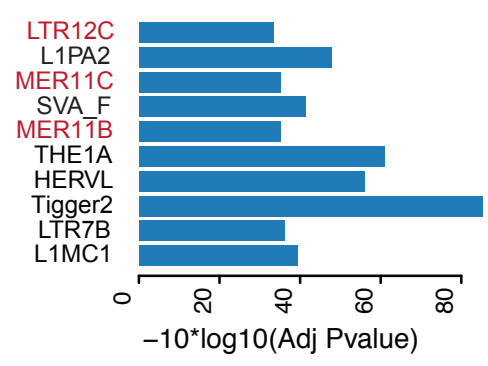

Hair color

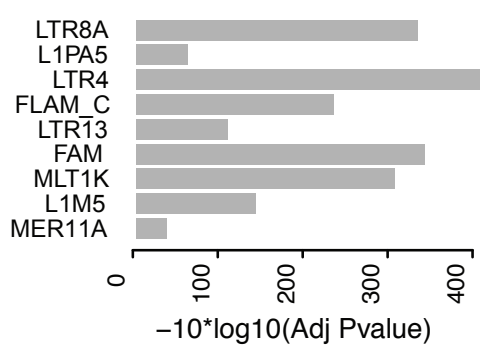

